\title{
Actual Issues FOR ReLEVANT LOGICS
}

\author{
SHAWN STANDEFER \\ School of Historical and Philosophical Studies, \\ The University of Melbourne
}

In this paper, I motivate the addition of an actuality operator to relevant logics. Straightforward ways of doing this are in tension with standard motivations for relevant logics, but I show how to add the operator in a way that permits one to maintain the intuitions behind relevant logics. I close by exploring some of the philosophical consequences of the addition.

$\mathrm{I}^{\mathrm{T}}$ $\mathrm{T}$ is common enough in work on relevant logics using Routley-Meyer frames to talk about a real world. This is naturally construed as the formal representative of the actual world. It is a natural step, though so far untaken, to add an actuality operator, $\mathbb{A}$, to provide a way to incorporate this idea into the object language for relevant logics. Some straightforward ways of doing this are, surprisingly, incompatible with the guiding intuitions behind relevant logics. In this paper, I will explore options for adding an actuality operator to relevant logics and some of the consequences of doing so.

In addition to the inherent logical interest of adding an actuality operator to relevant logics, there are some philosophical consequences of this work that are worth noting. There is much work on two-dimensional semantics and metaphysics developed against the background of classical modal logic. If one wants to explore the idea that a relevant logic can be one's general purpose logic, then there is a natural opening for developing non-classical semantic and metaphysical theories. Yet, without the groundwork developed here, one cannot begin to pursue twodimensional semantics and metaphysics against a relevant logical background. As we will see in the final section of this paper, there are substantive questions about how to develop such theories. Even if one is not a proponent of relevant

Contact: Shawn Standefer <sstandefer@unimelb.edu.au> 
logics, one might be interested in the development of non-classical metaphysics for the distinctions that it draws, which may pass unnoticed in the more familiar classical setting.

The picture of actuality that results in the context of relevant logics differs from the more familiar, classical two-dimensional picture in a number of ways. First, while in the classical setting of S5 there is a natural candidate for the logic of actuality, no similarly overwhelmingly natural candidate emerges in the relevant setting. There are some plausible candidate logics, but choosing among them requires further work. Second, it will emerge that the natural view of actuality for the relevant logician, and the relevance-inclined metaphysician, does not fit neatly with either an indexical or an absolute view of actuality. Finally, it raises some issues for actualism in ontology, as it complicates the notion of being an actual object.

The paper will begin with a brief introduction to relevant logics (§1). In §2, I will motivate the addition of an actuality operator, $\mathbb{A}$, to relevant logics. In $\$ 3$, I will argue against defining an actuality operator out of the resources available in standard relevant logics. Then I will examine two natural ways of interpreting actuality on Routley-Meyer frames and argue that they are not satisfactory (\$4). In $\S 5$, I will propose a new way to interpret the actuality operator that fits with relevant logics and discuss options for strengthening the basic logic. Following that, I will discuss some of the philosophical issues that arise from my proposal (§6). Finally, in the Appendix I will prove some of the technical results that arise in the course of the discussion.

\section{Introduction to Relevant Logics}

In this section, I will provide the basic background on relevant logics, their frame semantics, and axiom systems. Relevant logics are non-classical logics requiring a strong connection between the antecedent and consequent of valid conditionals. ${ }^{1}$ Part of the guiding intuition of relevant logics, at least in the early development by Anderson and Belnap (1975), was that the antecedent and consequent of a valid conditional had to be relevant to each other, in the sense that one had to really use the antecedent in obtaining consequent. ${ }^{2}$ While the exact demarcation of the connection was left somewhat open, a necessary condition on the connection

1. See Dunn and Restall (2002) or Bimbó (2006) for reader-friendly overviews of relevant logics.

2. How to understand the connection at issue is a delicate matter. Anderson and Belnap (1975) suggest a kind of connection based on use. An alternative conception is the sufficiency view of Routley (1977: 895ff.). A different approach to relevance is offered by Tennant (2017). 
is that the two formulas share a propositional variable. ${ }^{3}$ Both $p \rightarrow(q \rightarrow p)$ and $p \rightarrow(q \rightarrow q)$ exemplify the sort of irrelevance rejected by relevant logics; in the former, $q$ appears to be idle and in the latter, $p$ has nothing much to do with $q \rightarrow q$. Relatedly, implications in relevant logics are stricter than the material conditional, defined out of negation and disjunction, and so principles that are "too material" will also be rejected, such as $(p \wedge q) \rightarrow(p \rightarrow q) .4$ The fact that $p$ and $q$ are true together, accidentally as it were, falls short of establishing that $p$ implies $q$, in the sense of $p \rightarrow q$. These guiding intuitions will play a role in the later sections of this paper.

One of the main features of relevant logics is that they reject the axiom of Weakening, $A \rightarrow(B \rightarrow A)$, and its kin. ${ }^{5}$ For the purposes of this paper, a relevant logic is a logic between the logics $B$ and $R$, which will be defined shortly. Much of the focus of the paper will be on the Routley-Meyer frame semantics for relevant logics, although I will provide axioms for some logics under consideration below. ${ }^{6}$ The basic logics will be in the vocabulary $\{\rightarrow, \wedge, \sim, t\}$, with formulas built out of these connectives and a countable set of atoms, At. I will adopt the usual definitions for disjunction, $A \vee B={ }_{D f} \sim(\sim A \wedge \sim B)$, and the biconditional, $A \leftrightarrow B={ }_{D f}(A \rightarrow B) \wedge(B \rightarrow A)$. To cut down on parentheses, unary connectives will bind tightest, followed by conjunction and disjunction, which bind more tightly than the conditional and biconditional. The language will be extended with a unary operator, $\mathbb{A}$, later.

\subsection{Frames}

In this subsection, I will present Routley-Meyer frames for a range of relevant logics before providing axioms for them. The frames have a non-empty set of points, $\mathrm{K}$, and a distinguished subset $\mathrm{N} \subseteq \mathrm{K}$. The points are not best viewed as worlds, which settle too much. ${ }^{7}$ Rather, it is good to view them as restricted

3. This holds at least when sentential constants aren't in the language. When sentential constants, such as $\mathrm{t}$ below, are around then caveats are needed, but I will leave those on the sidelines for this paper.

4. Some work in the area draws a distinction between 'implication' and 'conditional', but I will use the terms interchangeably.

5. I will leave somewhat open the extent of the kindred axioms and rules. The particular demarcation adopted here excludes, e.g., R-Mingle, which is sometimes classed as a relevant logic. R-Mingle is obtained by adding the mingle axiom $A \rightarrow(A \rightarrow A)$ to $R$. Its status as a relevant logic is disputed on the grounds that it has theorems violating the variable sharing condition, such as $\sim(A \rightarrow A) \rightarrow(B \rightarrow B)$. See Avron (1992) for a discussion of R-Mingle and a defense of its intensional fragment.

6. Routley-Meyer frames were introduced by Routley and Meyer (1972a; 1972b; 1973). See Restall (2000: 235-274) for more recent developments in frame semantics. Routley changed his name to Sylvan later in life. I will cite his work by the published name.

7. This is notwithstanding the quotations to follow, which refer to some of the points as worlds. I will note that Mares and Meyer (1993) and Sedlár (2015) provide ways to define 
situations, which can be partial or contradictory. The normal points, $\mathrm{N}$, are the ones in which the logical truths hold, and the non-normal points, $K \backslash N$, are ones in which even logical truths can fail. Further discussion of the interpretation of the frames will be left till $\S 6$. The frames also have a ternary relation, $R$, that will be used to interpret the conditional and an operation, the Routley star, that will be used to interpret negation. ${ }^{8}$

The heredity ordering, $\leq$, is defined as $a \leq b={ }_{D f} \exists x \in N$, Rxab, and it will be important below. One can think of it as a containment relation. On the interpretation taking points to be situations, it would be the relation of one situation being contained in a bigger situation.

Definition 1. A Routley-Meyer frame, $\mathrm{F}$ is a quadruple $\left\langle\mathrm{K}, \mathrm{N},{ }^{*}, \mathrm{R}\right\rangle$, where $\mathrm{K} \neq \varnothing$, $\mathrm{N} \subseteq \mathrm{K},{ }^{*}: \mathrm{K} \mapsto \mathrm{K}$ such that $\mathrm{a}^{* *}=\mathrm{a}$, and $\mathrm{R} \subseteq \mathrm{K}^{3}$, satisfying the following conditions.

- $a \in N \wedge a \leq b \Rightarrow b \in N$.

- $\leq$ is reflexive and transitive.

- $a \leq b \Rightarrow b^{*} \leq a^{*}$.

- $\mathrm{d} \leq \mathrm{a} \wedge \mathrm{Rabc} \Rightarrow \mathrm{Rdbc}$.

One can narrow the class of frames by imposing frame conditions. Some standard ones are provided below, all of which should be read as their universal closures. Some of these conditions appeal to the standard definitions, Rabcd $=_{\mathrm{D} f}$ $\exists x(\operatorname{Rabx} \wedge \mathrm{R} x c d)$ and $R a(b c) d={ }_{D f} \exists x(\operatorname{Raxd} \wedge R b c x)$.

(F1) $\operatorname{Rabc} \Rightarrow \operatorname{Ra}(\mathrm{ab}) \mathrm{c}$

(F2) $\mathrm{Rabcd} \Rightarrow \mathrm{Rb}(\mathrm{ac}) \mathrm{d}$

(F3) $\mathrm{Rabcd} \Rightarrow \operatorname{Ra}(\mathrm{bc}) \mathrm{d}$

(F4) $\mathrm{Rabc} \Rightarrow$ Rabbc
(F5) $\mathrm{Rabc} \Rightarrow \mathrm{Rbac}$

(F6) Raaa

(F7) $R a b c \Rightarrow R a c^{*} b^{*}$

(F8) $\operatorname{Raa}^{*} a$

A Routley-Meyer model $M$ is a pair $\langle F, V\rangle$, where $F$ is a Routley-Meyer frame and $V: A t \mapsto \wp(K)$ is a valuation function on atoms such that if $a \leq b$ and $a \in V(p)$, then $b \in V(p)$, for all $p \in A t$. The valuation is extended to the whole language in the following way.

- $a \Vdash p$ iff $a \in V(p)$

- $\mathrm{a} \Vdash \mathrm{t}$ iff $\mathrm{a} \in \mathrm{N}$

worlds as distinguished points.

8. See Beall et al. (2012) for a discussion of the interpretation of the ternary relation. See Restall (1999) for a discussion of the interpretation of the Routley star. 
- $a \Vdash \sim A$ iff $a^{*} \Downarrow A$

- $a \Vdash A \wedge B$ iff $a \Vdash A$ and $a \Vdash B$

- $a \Vdash A \vee B$ iff $a \Vdash A$ or $a \Vdash B^{9}$

- $\mathrm{a} \Vdash \mathrm{A} \rightarrow \mathrm{B}$ iff $\forall \mathrm{y} z(\operatorname{Ray} z \wedge \mathrm{y} \Vdash \mathrm{A} \Rightarrow z \Vdash \mathrm{B})$

A model $M$ is built on the frame $F$ iff $M=\langle F, V\rangle$, for some $V$. A formula $A$ is true in a model $M$ iff for all $a \in N, a \Vdash A$. A formula $A$ is valid in a frame $F$, iff $A$ is true in all models $M$ built on $F$. A formula is valid in a class of frames $\mathcal{C}$ iff for all $F \in \mathcal{C}$, $A$ is valid on $F$. A set $X$ of formulas is valid in a class of frames $\mathcal{C}$ iff for all $A \in X$, $A$ is valid in the class. Where a formula $A$ is valid in a class of frames $\mathcal{C}$, I will write $\models_{\mathcal{C}} A$.

An important, standard lemma is that preservation along the heredity ordering holds for all formulas, not just atoms.

Lemma 1 (Heredity Lemma). If $\mathrm{a} \leq \mathrm{b}$ and $\mathrm{a} \Vdash \mathrm{A}$, then $\mathrm{b} \Vdash \mathrm{A}$.

Proof. The proof is by induction on the complexity of the formula A. See Read (1988: Ch. 5, §4) for an example of the argument.

The Heredity Lemma has the following consequence, which simplifies some later proofs.

Lemma 2 (Verification Lemma). Given a Routley-Meyer model $M, A \rightarrow B$ is true in $M$ iff $\forall x \in K(x \Vdash A \Rightarrow x \Vdash B)$.

Proof. $(\Rightarrow)$. Suppose $A \rightarrow B$ is true in $M$ and for some $a \in K, a \Vdash A$ but a $\forall$ B. As $a \leq a$, for some $b \in N$, Rbaa, but then $b \| A \rightarrow B$, contradicting the assumption.

$(\Leftarrow)$. Suppose $\forall x \in K(x \Vdash A \Rightarrow x \Vdash B)$ but $A \rightarrow B$ is not true in $M$. Then there are $a \in N$ and $b, c \in K$ such that Rabc, $b \Vdash A$, and $c \mid \forall B$. By the Heredity Lemma, $c \Vdash A$, but this contradicts the assumption.

Appeal to these two lemmas will be left implicit throughout. Let us now turn briefly to axioms.

\subsection{Axiom Systems}

In this subsection, I will present some Hilbert-style axiom systems for some common relevant logics. While the axiomatics are important for the technical results, the philosophical issues focus mostly on the frames, so the reader primarily concerned with the philosophical issues should feel free to skip this subsection.

9. Disjunction is defined, so this clause is, technically, a lemma rather than a stipulation. 
As is common for the area, the focus will be on logics as sets of theorems obtained by specification of axioms and rules. ${ }^{10}$ The basic relevant logic $B$ has the following axioms and rules. ${ }^{11}$ Put more carefully, $B$ is the least set of formulas containing all instances of the following axioms and closed under the following rules.
(A1) $A \rightarrow A$
(A8) $\mathrm{t}$
(A2) $\mathrm{A} \wedge \mathrm{B} \rightarrow \mathrm{A}, \mathrm{A} \wedge \mathrm{B} \rightarrow \mathrm{B}$
(R1) $\mathrm{A}, \mathrm{A} \rightarrow \mathrm{B} \Rightarrow \mathrm{B}$
$(\mathrm{A} 3)(\mathrm{A} \rightarrow \mathrm{B}) \wedge(\mathrm{A} \rightarrow \mathrm{C}) \rightarrow(\mathrm{A} \rightarrow \mathrm{B} \wedge \mathrm{C})$
(R2) $A, B \Rightarrow A \wedge B$
(A4) $\mathrm{A} \rightarrow \mathrm{A} \vee \mathrm{B}, \mathrm{B} \rightarrow \mathrm{A} \vee \mathrm{B}$
(R3) $\mathrm{A} \rightarrow \mathrm{B} \Rightarrow(\mathrm{B} \rightarrow \mathrm{C}) \rightarrow(\mathrm{A} \rightarrow \mathrm{C})$
$(\mathrm{A} 5)(\mathrm{A} \rightarrow \mathrm{C}) \wedge(\mathrm{B} \rightarrow \mathrm{C}) \rightarrow(\mathrm{A} \vee \mathrm{B}) \rightarrow \mathrm{C}$
$(\mathrm{R} 4) \mathrm{A} \rightarrow \mathrm{B} \Rightarrow(\mathrm{C} \rightarrow \mathrm{A}) \rightarrow(\mathrm{C} \rightarrow \mathrm{B})$
$(\mathrm{A6}) \mathrm{A} \wedge(\mathrm{B} \vee \mathrm{C}) \rightarrow(\mathrm{A} \wedge \mathrm{B}) \vee(\mathrm{A} \wedge \mathrm{C})$
(R5) $A \rightarrow B \Rightarrow \sim B \rightarrow \sim A^{12}$
(A7) $\sim A \leftrightarrow A$
(R6) $A \Rightarrow t \rightarrow A$

Stronger logics can be obtained with the addition of axioms. The frame conditions of the previous section correspond to the following axioms, where axiom $(\mathrm{Bi})$ is valid in the class of frames obeying condition $(\mathrm{Fi}) .{ }^{13}$

$$
(\mathrm{A} \rightarrow \mathrm{B}) \wedge(\mathrm{B} \rightarrow \mathrm{C}) \rightarrow(\mathrm{A} \rightarrow \mathrm{C})
$$

$(\mathrm{B} 2)(\mathrm{A} \rightarrow \mathrm{B}) \rightarrow((\mathrm{B} \rightarrow \mathrm{C}) \rightarrow(\mathrm{A} \rightarrow \mathrm{C}))$

(B3) $(\mathrm{A} \rightarrow \mathrm{B}) \rightarrow((\mathrm{C} \rightarrow \mathrm{A}) \rightarrow(\mathrm{C} \rightarrow \mathrm{B}))$

(B4) $(\mathrm{A} \rightarrow(\mathrm{A} \rightarrow \mathrm{B})) \rightarrow(\mathrm{A} \rightarrow \mathrm{B})$
(B5) $\mathrm{A} \rightarrow((\mathrm{A} \rightarrow \mathrm{B}) \rightarrow \mathrm{B})$

(B6) $(\mathrm{A} \wedge(\mathrm{A} \rightarrow \mathrm{B})) \rightarrow \mathrm{B}$

(B7) $(\mathrm{A} \rightarrow \mathrm{B}) \rightarrow(\sim \mathrm{B} \rightarrow \sim \mathrm{A})$

(B8) $(\mathrm{A} \rightarrow \sim \mathrm{A}) \rightarrow \sim \mathrm{A}$

The following well known logics can be obtained by the addition of the indicated axioms, with $B$ being $(A 1)-(A 8)+(R 1)-(R 6) .{ }^{14}$

10. The logical framework adopted here is FMLA, rather than one of the more general consequence relation frameworks, e.g., SET-FMLA. See Humberstone (2011: 103ff.).

11. The symbol ' $\Rightarrow$ ' does double duty as the metatheoretic material conditional and the separator between premises and conclusion of the rules of the object logic.

12. The rule $A \rightarrow \sim B \Rightarrow B \rightarrow \sim A$ is commonly used instead of (R5). This rule can be obtained from (R5), and vice versa, given the other rules and axioms.

13. For the correspondence results, which are standard, see Routley, Plumwood, Meyer, and Brady (1982: Ch. 4).

14. These are somewhat redundant axiomatizations. For example, (R5) is redundant once (B7) is added, and (B6) follows from (B4). See Brady (1984), on which this presentation is based, for non-redundant axiomatizations. 
- $\mathrm{DW}=\mathrm{B}+(\mathrm{B} 7)$

- $\mathrm{DJ}=\mathrm{DW}+(\mathrm{B} 1)$

- $\mathrm{TW}=\mathrm{DW}+(\mathrm{B} 2)+(\mathrm{B} 3)$
- $\mathrm{T}=\mathrm{TW}+(\mathrm{B} 4)+(\mathrm{B} 6)+(\mathrm{B} 8)$

- $\mathrm{RW}=\mathrm{TW}+(\mathrm{B} 5)$

- $\mathrm{R}=\mathrm{RW}+(\mathrm{B} 4)+(\mathrm{B} 6)+(\mathrm{B} 8)$

The results in this paper are largely independent of the particular base logic, provided the logic falls between $B$ and $R$.

A formula $A$ is provable in the axiomatic system for the logic $L, \vdash_{L} A$, iff there is a finite sequence of formulas, each of which is either an axiom or is the conclusion of a rule whose premises are earlier members of the sequence, ending in $A$. Such a formula is a theorem of $L$. The logic $L$ is the set of formulas $A$ such that $r_{\mathrm{L}} A$.

Say that $\mathcal{F}(L)$ is the class of frames satisfying the frame conditions for the axioms of $L$. A logic $L$ is sound with respect to a class of frames $\mathcal{C}$ iff for all formulas $A, \vdash_{L} A$ only if $\models_{\mathcal{C}} A$. A logic $L$ is complete with respect to a class of frames $\mathcal{C}$ iff for all formulas $A, \models_{\mathcal{C}} A$ only if $\vdash_{L} A$.

Theorem 1. The logic $\mathrm{B}$ is sound and complete with respect to the class of all RoutleyMeyer frames, $\mathcal{F}(\mathrm{B})$. The logic $\mathrm{L}$ obtained by adding axioms from (B1)-(B8) is sound and complete with respect to the class of frames obeying the corresponding frame conditions.

Proof. See Restall (200o: Ch. 11) for an example of the Henkin-style canonical model proof technique.

Let us proceed to motivations for adding an actuality operator to relevant logics.

\section{Actuality}

As mentioned at the outset, it is not hard to find examples, in the relevant logic literature, of people talking about the real, or actual, world in Routley-Meyer frames. ${ }^{15}$ This is particularly the case in early work on Routley-Meyer frame semantics, where the frames were slightly different. An RMg-frame is a tuple $\left\langle K, N, g,{ }^{*}, R\right\rangle$ where $\left\langle K, N,{ }^{*}, R\right\rangle$ is a Routley-Meyer frame and $g \in N .{ }^{16}$ Let us look at some quotations, in which emphasis has been added. We begin at the beginning, with the early work of Routley and Meyer.

15. There has been comparatively little written on actuality in non-classical logics. A near relative is the work on intuitionistic hybrid logic of Braüner and de Paiva (2006) and Braüner $(2006 ; 2007 ; 2011)$, as well as the work on paraconsistent hybrid logic of Costa and Martins (2017).

16. In the early work, such as Routley and Meyer (1973), there was no set N. Instead, g did the work of defining the heredity ordering and being used to define truth in a model. The distinguished world, $\mathrm{g}$, was sometimes denoted by ' $\mathrm{o}$ '. 
The real world [g] plays a distinguished role in our semantical postulates. (Routley \& Meyer 1973: 202)

A final word concerns the role of [g] in the semantics of this paper as contrasted with its predecessors and successor. [The set-up g] is a preferred set-up in which all logical truths hold, according to [Routley and Meyer (1973)]. This remains true. But in [Routley and Meyer (1973)] and [Routley and Meyer (1972a)], [g] could also be thought of as a normal set-up - a candidate, so to speak, to be the 'real' world, in that each sentence or its negation could be made to turn out true in [g] without ever making both true; logical truth then turns out to be truth in all 'real' worlds. Thus [g] has a regulative role - the logical truths are true there - and a normal one. (Routley \& Meyer 1972b: 197)

Since the introduction of Routley-Meyer models, there have been questions about the philosophical interpretation of the models. As indicated by the preceding quotations, in their early papers, Routley and Meyer talked about the distinguished world as the real world. This is naturally taken to be a candidate for representing the actual world, although they do not put it in quite those terms, and they did not add an operator to refer to that world in the object language. In slightly later work with Plumwood and Brady, they call the distinguished world "factual", again suggesting it be taken to represent the actual world. ${ }^{17}$

The only world that determines truth is the factual one, [g]. Other worlds need not resemble the factual world in any way, and what holds in them does not provide some simulacrum of truth, nor, in terms of what these are about, of existence. (Routley et al. 1982: 294)

It was not just Routley and Meyer who talked about the real world of the models. Other authors, such as Slaney and Giambrone, did as well. Slaney and Giambrone are discussing reduced models, which require a bit of explanation. In this paper, I have adopted a form of Routley-Meyer model that has became standard, one that has a set of normal worlds, $\mathrm{N}$, rather than just a single normal world, $\mathrm{g}$. Reduced models are those which adhere more closely to the original presentation of Routley-Meyer models, using singleton sets of normal worlds rather than a potentially larger set of normal worlds. Truth in a model is defined with respect to the single normal world of the model. These models have some technical niceties. As indicated by the following quotations, there is also a philosophical viewpoint that seems allied to the reduced models. On this view, the single normal world of each model represents the real world, with the rest of the worlds doing the semantic work of ensuring that the conditionals are properly relevant.

17. The "factual world" terminology is also used by Routley (1984: 149). 
It is splendid that the main contractionless relevant systems allow the possibility of models in which there are no contingent truths, for logic is in a way impure when it claims there are truths about matters which are not its business; but it is also desirable that there be models looking like reality, in which there are many truths beyond what is given by Pure Reason. (Slaney 1987: 406)

Giambrone (1992) responds to Slaney (1987), but there is no disavowal of the idea that the distinguished world is the real one. Indeed, Giambrone reiterates the point.

Slaney has provided reduced models (ones in which there is but one "real" world) ... (Giambrone 1992: 442)

In particular, the real worlds of our "real" models are large in that appropriate if somewhat obscure sense of [Slaney (1987)]: there really are "models looking like reality, in which there are many truths beyond what is given by Pure reason". (Giambrone 1992: 442-447)

The final quotation, from Fuhrmann, is particularly of interest because he had, at the time of publication, recently finished his $\mathrm{PhD}$ thesis at ANU, which was then a hotbed of relevant logic activity. ${ }^{18} \mathrm{I}$ take it that the intuition he describes is one that was in the air at the time.

The models introduced in Section II are not the, in a sense, preferred models for relevant logics. It is sometimes thought to be intuitively more satisfactory, if the set $[\mathrm{N}]$ of "distinguished worlds" could be reduced to a singleton set containing just the "real world", [g]. In such a reduced model a sentence is true (in a model) just in case it is true in the real world (of that model). (Fuhrmann 1990: 510)

These quotations, taken together, give an indication that the idea that the distinguished world does, or at least can, represent the actual world was relatively common in work on relevant logics. ${ }^{19}$ The idea that a model can contain a distinguished world that represents the actual world has been studied in the context of

18. Sylvan, Meyer, and Slaney were working at ANU, and Slaney, Giambrone, and Fuhrmann were all doctoral students there. In addition, there were regular visits by other logicians from around Australasia working in the relevant logic tradition.

19. While these quotations call the points 'worlds', they are often not regarded as such in the literature on relevant logics, as worlds settle too much. I will come back to the interpretation of the points in $\S 6$. 
classical modal logic. ${ }^{20}$ There are no formal problems with talking about actuality, via a unary operator, $\mathbb{A}$, in classical modal logic. ${ }^{21}$ The logic for actuality, S5A, of Crossley and Humberstone (1977) adds the following axioms and rule to classical $\mathrm{S} 5$, with the rule (Nec), from $A$ to infer $\square A$.

- $\mathbb{A}(\mathbb{A} A \supset A)^{22}$

- $\mathbb{A}(A \supset B) \supset(\mathbb{A} A \supset \mathbb{A B})$

- $\mathbb{A} A \equiv \sim \mathbb{A} \sim A$

- $\square \mathrm{A} \supset \mathbb{A A}$

- $\mathbb{A} A \supset \square \mathbb{A} A$

The frames for this logic add a distinguished world, g, to a Kripke frame with a universal accessibility relation. The axioms for $\mathbb{A}$ are enough to ensure that the operator commutes with the truth-functional connectives. This can be reproduced in the relevant logical context, although there appear to be some options as to what the logic should be, which I will explore in $\S 5$ and §6.2.

In addition to there being no formal barrier to studying actuality in classical modal logic, there are many benefits from having such an operator. ${ }^{23}$ The addition of actuality to classical modal logics connects to the rich field of two-dimensional semantics. ${ }^{24}$ Apart from the attempt to formalize some of the ideas from the motivating quotations above, there would be value in taking steps towards nonclassical, and specifically relevant, theories of actuality and two-dimensional semantics. ${ }^{25}$

20. Kripke (1963: 69) calls the distinguished world of the models the "real world". This usage is not restricted to classical modal logic or Routley-Meyer frames. See, e.g., Priest (2006: 85-87), where the distinguished world of the model is described as "the 'real world' or assignment which is in accord with the actual" and this idea is used to argue in favor of certain properties of the distinguished world.

21. See, e.g., Crossley and Humberstone (1977), Hazen, Rin, and Wehmeier (2013), Gilbert and Mares (2012), or Fritz (2013; 2014). For proof-theoretic approaches to actuality, see Restall (2012), Gilbert (2016), and Lampert (2018a).

22. Davies and Humberstone (1980) note that this axiom is redundant, given the rest of the axioms and rules. It does not, however, follow from the other actuality axioms with the addition of a ( $\mathrm{Nec})$ rule for actuality. As it is somewhat distinctive for actuality, I include it here. I thank Fabio Lampert for drawing my attention to the redundancy.

23. See, e.g., Davies and Humberstone (1980), Humberstone (2004), and Lampert (2018b).

24. See Schroeter (2017) for an overview.

25. There are further related topics in the area that would be worth exploring. Wehmeier (2004) shows an equivalence between classical S5 with actuality and a modal language with subjunctive predicates in addition to regular predicates. Would such an equivalence hold in the relevant logical context or would there be some expressive difference between languages with subjunctive atoms and those without subjunctive atoms but with an actuality operator? French 
The question I will explore for the remainder of the paper is how one can make good on the idea of talking about actuality, formally, through the addition of an actuality operator to relevant logics while maintaining something of the relevance intuitions that many find attractive about relevant logics. I will consider three options before settling on the preferred approach of this paper. For the remainder of the paper, the language will include a unary operator, $\mathbb{A}$.

\section{Why Not the Ackermann Constant?}

The first option eschews the addition of anything new to the familiar frames in favor of trying to define actuality in terms of the Ackermann constant, t. The intuitive interpretation of $\mathrm{t}$ is the conjunction of all logical truths (Anderson \& Belnap 1975: 342). The interpretation of $t$ in a model is the set $N$ of normal worlds, worlds at which the truths of logic hold. Plausibly, the actual world is normal in that sense, so one might think that there could be a connection between an actuality operator and $t$. This approach would offer one main benefit over the other approaches to be considered. That benefit is that it does not require the addition of new logical machinery, instead using the rich resources of relevant logics to define the new operator. One would simply adopt an axiom, $\mathbb{A} A \leftrightarrow \phi(A)$, where $\phi$ is some propositional context involving $t$. At least, that would be the benefit, were this approach to work out.

There are two natural candidates for defining $\mathbb{A}: t \wedge_{-}$and $t \rightarrow_{-}$. I will take these in turn. The conjunctive version has some apparently unpalatable consequences. For example, it has the consequence that $\mathbb{A p} \rightarrow(q \rightarrow q)$ is valid in the class of all RM-frames. It is perfectly understandable and welcome that $\mathrm{t} \wedge \mathrm{p} \rightarrow(\mathrm{q} \rightarrow \mathrm{q})$ is valid, as $\mathrm{t} \wedge \mathrm{p} \rightarrow \mathrm{t}$ and $\mathrm{t} \rightarrow(\mathrm{q} \rightarrow \mathrm{q})$ are both theorems of $\mathrm{B}$. It is, however, intuitively wrong that it actually being the case that $p$ should imply, in the strong sense of the relevant conditional, any old logical truth, such as $q \rightarrow q$. This greatly strains-if not outright violates-relevance intuitions. After all, $p$ does not imply $q \rightarrow q$, and the addition of 'actually' does not seem to establish the appropriate sort of connection between $p$ and $q \rightarrow q$. I will, then, set the conjunctive option aside.

The conditional form, taking $\mathbb{A} A$ to be $t \rightarrow A$, does better on the relevance intuitions front, but the philosophical interpretation is off. The Ackermann constant, $t$, is the minimal logical truth, the conjunction of all logical truths for the particular logic under consideration. As a definition of actuality, being implied by pure logic seems dubious. Indeed, this just seems to be the wrong approach to contingent actual facts. Such contingencies might true along with, but not be

(2015) shows that a difference emerges in between the languages when a classical modal logic weaker than S5 is used, so it seems likely that differences will emerge against a relevant logical background. I thank Fabio Lampert for this suggestion and Rohan French for discussion. 
implied by the truths of logic, as the negations of those contingencies should also, at least potentially, be true along with the truths of logic, without being implied by them. Adapting a phrase from Slaney, such contingencies seem not to be logic's business.

These points remain at the level of intuition, but the intuitions against the conditional definition of actuality can be strengthened. There are several principles that one may want to adopt to strengthen the logic of actuality, such as $\sim \mathbb{A} A \leftrightarrow \mathbb{A} \sim A$ or $\mathbb{A}(A \vee B) \leftrightarrow \mathbb{A} A \vee \mathbb{A} B$. When unpacked using the definition of ' $\mathbb{A}^{\prime}$, we get $\sim(t \rightarrow A) \leftrightarrow(t \rightarrow \sim A)$ and $(t \rightarrow(A \vee B)) \leftrightarrow(t \rightarrow A) \vee(t \rightarrow B)$, respectively. The latter will be in inappropriate in any logic, such as $T$, in which excluded middle is a theorem but $(t \rightarrow A) \leftrightarrow A$ is not, on pain of $(t \rightarrow B) \vee(t \rightarrow \sim B)$ being valid for every formula, where $t$ need not imply either $B$ or $\sim B$, for contingent $B$. Even in weaker logics, these principles are, at best, unusual for the operator "implied by logic" whereas they are fine for the logic of actuality. The interpretation of $t$ is tied to the overall logic, while there are additional axioms that are plausible for governing just actuality.

When $(t \rightarrow A) \leftrightarrow A$ is a theorem of the logic, as is the case with $R$, the distinction between $\mathbb{A} A$ and $A$ collapses, as a matter of logic. Yet, it seems plausible to maintain that logic should not collapse that distinction, that logic should deal with what is sometimes called general validity, as opposed to real-world validity. ${ }^{26}$ At the very least, which of the two forms of validity we opt for should be a choice. So, I conclude that the proposed conditional definition, $t \rightarrow_{-}$, is out as a definition of "actually". ${ }^{27}$

The objections to both options share a common core: The options are both bad things to mean by "actually". Both options define operators that may be interesting in their own right. For example, the implicational definition provides a kind of logical necessity, along the lines discussed by Anderson and Belnap (1975: Ch.1). Neither option, however, provides a plausible, attractive thing to

26. See Davies and Humberstone (1980), Zalta (1988), Humberstone (2004), Hanson (2006), Nelson and Zalta (2012), and French (2012) for discussion.

27. There is a third option for defining actuality that was not included above, since it involves a connective that is not primitive in the language, namely fusion, $\circ$. If we add fusion to the language, then we also need to add the rules $A \rightarrow(B \rightarrow C) \Rightarrow(A \circ B) \rightarrow C$ and $(A \circ B) \rightarrow C \Rightarrow A \rightarrow(B \rightarrow C)$. A natural way to attempt to define an actuality operator using fusion is as $t \circ \ldots$. The problem with this is that it makes $A \leftrightarrow(t \circ A)$ valid, even in $B$. To see this, note that $t \rightarrow(A \rightarrow A)$ is a theorem of $B$, which by one fusion rule yields $(t \circ A) \rightarrow A$. For the other direction, we have $t \rightarrow(A \rightarrow(t \circ A))$ by $(A 1)$ and a fusion rule. From (A8) and (R1), we then have $A \rightarrow(t \circ A)$. As with the implicational definition of actuality, whether $A A \leftrightarrow A$ is valid is not something that the underlying logic should settle.

In weaker logics, $t \circ A$ is not equivalent to $A \circ t$, suggesting a potential option for defining actuality as _o t. This option is a non-starter, as it would result in $\mathbb{A} A \wedge \mathbb{A B} \rightarrow \mathbb{A}(A \wedge B)$ being invalid in $T$ and weaker logics. I would like to thank an anonymous referee for asking about these potential definitions of the actuality operator. 
mean by "actually", although their faults are different. Let us then continue to the next candidate.

\section{Distinguished World}

The second approach to adding actuality is to straightforwardly adapt the double indexing approach to actuality in classical modal logic as found in the RMgframes. Evaluation of a formula will be done at a pair of points, $\langle g, a\rangle$, where $g$ is the distinguished world of the frame, which I will assume is in N. The truth conditions for actuality on this approach are as follows.

- $g, a \Vdash \mathbb{A} A$ iff $g, g \Vdash A$

Double indexing is adopted here to facilitate some later discussion and connections to other work in two-dimensional semantics. For the point of the present section, one could keep single indexing, with the actual point $g$ distinguished by the frame, and adapt the truth condition to use only the righthand point.

One needs to check that the Heredity Lemma holds in the extended language. In particular, let us check the new case, for formulas of the form $\mathbb{A B}$.

Proof. Suppose $\mathrm{a} \leq \mathrm{b}$ and $\mathrm{g}, \mathrm{a} \Vdash \mathbb{A B}$. So, $\mathrm{g}, \mathrm{g} \Vdash \mathrm{B}$. Therefore, $\mathrm{g}, \mathrm{b} \Vdash \mathbb{A B}$.

The lemma, then, holds for the extended language, but the short proof above should raise a red flag: The heredity assumption was not needed. This suggests that irrelevancy is afoot. Let us make good on that suggestion.

Lemma 3 (Ubiquity Lemma). For all $R M g$-models, for all $\mathrm{a}, \mathrm{b} \in \mathrm{K}$, if $\mathrm{g}, \mathrm{a} \Vdash \mathbb{A} A$, then $\mathrm{g}, \mathrm{b} \Vdash \mathbb{A} A$.

Proof. Let $M$ be a RMg-model and suppose $g, a \Vdash \mathbb{A} A$. This is the case iff $g, g \Vdash A$, which is the case iff $\mathrm{g}, \mathrm{b} \Vdash \mathbb{A} A$, as desired.

The red flags have now been joined by warning klaxons. This leads, as one might expect, to many disastrous consequences, of which I will highlight four.

Proposition 1. In the class of all RMg-frames, the following are valid.

- $q \rightarrow \mathbb{A}(p \rightarrow p)$

- $\mathbb{A p} \rightarrow(q \rightarrow \mathbb{A p})$

- $\mathbb{A}(p \wedge q) \rightarrow(\mathbb{A} p \leftrightarrow \mathbb{A} q)$

- $\mathbb{A} p \wedge \sim \mathbb{A} p \rightarrow q$

Ergo • vol. 7, no. $8 \cdot 2020$ 
Proof. Let us begin with the first. Take a model on an arbitrary frame and suppose $g, a \Vdash q$. As $g \in N, g, g \Vdash p \rightarrow p$, so $g, a \Vdash \mathbb{A}(p \rightarrow p)$. Therefore, $q \rightarrow \mathbb{A}(p \rightarrow p)$ is valid.

For the second, take a model on an arbitrary frame, and suppose $g$, a $\Vdash \mathbb{A}$. We want to show $\mathrm{g}, \mathrm{a} \Vdash \mathrm{q} \rightarrow \mathbb{A}$. Suppose $\mathrm{b}$ and $\mathrm{c}$ are such that Rabc and $\mathrm{g}, \mathrm{b} \Vdash \mathrm{q}$. By lemma 3, g, c $\Vdash \mathbb{A} p$. Therefore, g, a $\Vdash \mathrm{q} \rightarrow \mathbb{A} p$, which suffices for the claim.

For the third, suppose $g, a \Vdash \mathbb{A}(p \wedge q)$. By simple argument, $g, a \Vdash \mathbb{A} p$ and $\mathrm{g}, \mathrm{a} \Vdash \mathbb{A}$. Suppose $\mathrm{b}$ and $\mathrm{c}$ are such that Rabc and $\mathrm{g}, \mathrm{b} \Vdash \mathbb{A}$. By lemma 3, $\mathrm{g}, \mathrm{c} \Vdash \mathbb{A} q$, which suffices for $\mathrm{g}, \mathrm{a} \Vdash \mathbb{A} p \rightarrow \mathbb{A} q$. The converse conditional is similar.

For the fourth, suppose it is not valid. Then there is a counterexample model with a point a such that $g, a \Vdash \mathbb{A} p \wedge \sim \mathbb{A} p$ but $g, a \forall q$. It follows that $g, a \Vdash \mathbb{A} p$, so by lemma $3, g, b \Vdash \mathbb{A}$, for all $b \in K$. It also follows from the assumption that $\mathrm{g}, \mathrm{a} \Vdash \sim \mathbb{A p}$, so $\mathrm{g}, \mathrm{a}^{*} \forall \mathbb{A}$, which is a contradiction. Thus, there is no counterexample.

It is clear, then, that the simple double indexing approach flagrantly violates relevance intuitions. Simple double indexing does not provide the sort of semantic wiggle room needed for models to falsify irrelevant conditionals. Indeed, this sort of problem may arise when new logical vocabulary is added to the basic relevant logical language. For example, suppose an identity predicate were in the language, interpreted at each point as the set of pairs $\langle 0,0\rangle$ for each object $o$ in the domain. Then, $i=i$ would be valid, but so would $p \rightarrow i=i$, despite the lack of connection between the antecedent and consequent. Some wiggle room is needed in order to falsify logical truths using the new vocabulary so that no irrelevancy is introduced. Fuhrmann (1990: 509) calls a formula $A$ true at all points of $M$ ubiquitous in $M$, following a suggestion of Humberstone. With the introduction of new vocabulary, one needs to avoid thereby generating ubiquitous truths. ${ }^{28}$ Simple double indexing introduces ubiquitous truths with abandon.

While a single point won't cut it to interpret actuality, one might think that several points would. For a given frame, a set of points $X$ is hereditarily closed iff if $a \in X$ and $a \leq b$, then $b \in X$. Sets that are hereditarily closed are propositions. ${ }^{29}$ Rather than a single point representing the actual world, perhaps one should instead take a proposition to represent how things actually are, with the actuality operator acting as a universal quantifier over that proposition. This move does not, in the end, make a great difference, as many of the problems highlighted above for a distinguished point can be reproduced. Yet more flexibility is required to prevent formulas of the form $\mathbb{A B}$ from being ubiquitous, if true.

28. Mares (1992) provides a way to model identity that does not generate ubiquitous truth and irrelevancy. The extension of the identity predicate is required to be reflexive over $\mathrm{N}$, but not elsewhere. Hence, $i=i$ will turn out valid while $p \rightarrow i=i$ can be invalidated.

29. In the Kripke frames for classical modal logic, arbitrary sets of points can be propositions. In the present setting, arbitrary sets of points will not do. 


\section{Differing Views on Actuality}

While the distinguished subset approach does not deliver an advance on the problems with the RMg-frames, it does provide a nice stepping stone to the solution adopted in this paper. The previous two options took the interpretation of actuality to be globally constant. It is that requirement that is too rigid. Rather than having a distinguished world or proposition, instead, I will opt for using a binary accessibility relation, $S$, to pick out what each point takes to be actual. ${ }^{30}$ The relation Sab holds iff a takes all actual truths to hold in $b$.

The interpretation of $S$ fits with an epistemic interpretation on the RM-frames, on which each point represents a body of information. The information may be incomplete and it may be inconsistent. Some, though perhaps not all, bodies of information talk about what is actually the case. Some of these points may be deeply, deeply confused about what is actual, but they can still take things to be actual, however wrong they turn out to be. It is this possibility, that different points can differ on what they take to be actual, that will provide the flexibility needed to break the disastrous ubiquity of actuality formulas of the previous section. In §6.1, I will return to the interpretation of the frames.

Where $\mathrm{S} \subseteq \mathrm{K} \times \mathrm{K}$, I will use the following defined notation, following Meyer and Mares (1993).

- $S a={ }_{D f}\{b \in K: S a b\}$

- $\mathrm{R}(\mathrm{Sa})(\mathrm{Sb}) \mathrm{c}==_{\mathrm{Df}} \exists \mathrm{u} \exists v(\mathrm{Ruvc} \wedge \mathrm{Sau} \wedge \mathrm{Sbv})$

- $S(R a b) c={ }_{D f} \exists x(\operatorname{Rabx} \wedge S x c)$

Let us call an RM-frame augmented with a relation $S \subseteq \mathrm{K} \times \mathrm{K}$ an RMS-frame, provided it obeys the following constraint.

- $\mathrm{a} \leq \mathrm{b} \Rightarrow \mathrm{Sb} \subseteq \mathrm{Sa}$

This constraint makes sense on the gloss given above for $S$, understanding $\leq$ to be a kind of containment relation. It says that as the points say more, they may rule out options for actuality, which seems plausible. ${ }^{31}$

The truth condition for actuality appropriate for RMS-frames is the following.

30. A similar idea was explored, in the classical modal setting, by Gregory (2001), with Blackburn and Marx (2002) providing further discussion and technical development. I thank Rohan French for pointing out Gregory's paper to me and Fabio Lampert for pointing out Blackburn and Marx's.

31. There is a second condition that is worth highlighting: $\mathrm{Sab} \wedge \mathrm{b} \leq \mathrm{c} \Rightarrow$ Sac. This condition is optional, unlike the previous constraint, and its addition does not affect the logic. Informally, it says that if a takes all actual truths to hold in $b$, which is contained in $c$, then $a$ takes all the actual truths to hold in c. 
- $a \Vdash \mathbb{A} A$ iff $\forall y($ Say $\Rightarrow y \Vdash A)$

Let us return again to the $A B$ case of the proof of the Heredity Lemma.

Proof. Suppose $\mathrm{a} \Vdash \mathbb{A} B$ and $\mathrm{a} \leq \mathrm{b}$. Suppose $\mathrm{b} \| \mathbb{A} \mathrm{B}$. So, there is some $\mathrm{c}$ such that $S b c$ and $c \| \forall$. As $a \leq b, S b \subseteq S a$, so Sac. But then, a $\| \mathbb{A} B$, contradicting the assumption.

This time, the heredity assumption was used in a substantive way. This suggests that the blatant irrelevancies of the earlier sections will be avoided in the present setting. This idea will be borne out at the end of the Appendix.

The class of RMS-frames validates the following principles concerning actuality, in addition to the principles of the underlying logic.

- $(\mathbb{A} \wedge) \mathbb{A A} \wedge \mathbb{A B} \rightarrow \mathbb{A}(A \wedge B)$

- $(\mathrm{RM}) \mathrm{A} \rightarrow \mathrm{B} \Rightarrow \mathbb{A} A \rightarrow \mathbb{A B}$

All the logics under consideration entail the converse of $(\mathbb{A} \wedge),(\mathbb{A} \wedge c)$, via $(R M)$, (A2), and (R2). I will use the convention that L.M will be the logic of actuality that adds to the axioms and rules of the underlying logic $L$ both $(\mathbb{A} \wedge)$ and $(R M)$, and L.MX will be L.M with the addition of the principles in X..$^{32}$ Table $I$ lists some of the better studied principles that may be appropriate for actuality with their frame conditions. 33
(D) $\mathbb{A} \sim A \rightarrow \sim \mathbb{A} A$
(FD) $\forall x \exists y\left(S x y^{*} \wedge S x^{*} y\right)$
(4) $\mathbb{A} A \rightarrow \mathbb{A} \mathbb{A} A$
(F4) $\forall x \forall y \forall z(S x y \wedge S y z \Rightarrow S x z)$
$(\mathrm{K}) \mathbb{A}(\mathrm{A} \rightarrow \mathrm{B}) \rightarrow(\mathbb{A A} \rightarrow \mathbb{A B})$
(FK) $\forall x \forall y \forall z(S(R x y) z \Rightarrow R(S x)(S y) z)$
$(\mathrm{Nec}) \mathrm{A} \Rightarrow \mathbb{A} A$
$(\mathrm{FNec}) \forall x(x \in \mathrm{N} \Rightarrow \mathrm{S} x \subseteq \mathrm{N})$

Table 1. Familiar axioms and their frame conditions

There are some additional principles one might want to adopt whose frame conditions for RMS-frames are not well studied. To make negation fully commute with the actuality operator, we need $(\mathrm{Dc}) \sim \mathbb{A} A \rightarrow \mathbb{A} \sim A$, whose frame condition is

$$
\text { (FDc) } \forall x \forall y \forall z\left(S x^{*} y \wedge S x z \Rightarrow y^{*} \leq z\right) .34
$$

The adoption of (D) and (Dc) is sufficient to ensure both that actuality commutes with disjunction and that negations flanking an actuality operator cancel out, as recorded in lemma 4 in the Appendix.

32. As an example, DW.MD is the logic obtained by adding to the axioms and rules of DW, i.e., $(A 1)-(A 8),(R 1)-(R 6)$, and $(B 7),(\mathbb{A} \wedge),(R M)$, and $(D)$.

33. These are from Fuhrmann (1990: 507-508). I will adopt the convention that for a modal axiom or rule $(A)$, the corresponding frame condition is (FA).

34. I will leave proofs of soundness results to $\$ 7$. 
Next, I will turn to the axiom $(U) \mathbb{A}(\mathbb{A} A \rightarrow A) .{ }^{35}$ This corresponds to the frame condition

$$
\text { (FU) } \forall x \in N, \forall y(S x y \Rightarrow \forall z \forall u(R y z u \Rightarrow S z u)) .
$$

In the context of the classical modal logic of Crossley and Humberstone (1977), the embedded converse, $(U c) \mathbb{A}(A \rightarrow \mathbb{A} A)$, is a consequence of the other axioms and rules, replacing the relevant implication with the classical material conditional. Provided the contraposition axiom, (B7), (U), and (Dc) are in the logic, then the embedded converse follows from the other principles, for which proof see the Appendix. If the logic is lacking these principles, then one can validate (Uc) manually, with the following frame condition.

$$
\text { (FUc) } \forall x \in N, \forall y(S x y \Rightarrow \forall u \forall v(R y u v \Rightarrow \forall z(S v z \Rightarrow u \leq z)))
$$

There are further principles that one could add, following Crossley and Humberstone (1977). Although many of these principles follow from the other axioms in the classical modal context, they may be independent in the relevant context. Rather than attempt an exhaustive catalog of axioms, I will turn to discussion of some philosophical issues. In the Appendix, I address some metatheoretic issues.

\section{Discussion}

In this section, I will discuss some of the philosophical issues arising from the preceding work. After a brief summary, which will diagnose the problem with ubiquity, I will begin by discussing the interpretation of the frames (\$6.1) and then highlight some natural candidates for a preferred logic of actuality (\$6.2). Following this, I will look at how the view presented here fits into two debates in metaphysics, namely the debate between indexical and absolute theories of actuality and the debate between actualism and possibilism in ontology (\$6.3). I will then discuss issues arising in the interplay between actuality and necessity, in particular concerns about the fixedly operator of Crossley and Humberstone (§6.4), before closing by briefly mentioning directions for future work (§6.5).

One might have thought that it would be no problem to add an actuality operator to relevant logics. Natural ways of extending the Routley-Meyer frames to accommodate the actuality operator lead to direct conflicts with relevance intuitions that motivate work in the area. As indicated in $\S_{4}$, the problems stem from the interpretation of actuality. In an RMg-model, the interpretation of actuality is constant across the model. This leads to true actuality formulas being ubiquitous in the models.

35. The name is taken from Humberstone (2016: 33). 
The problems with the RMg-frames were solved by moving to RMS-frames. The cost of RMS-frames is that one adopts a somewhat removed view of the actual world in the sense that the points need not all agree on which world, or proposition, is actual. The Ubiquity Lemma suggests why global agreement on what is actual will be difficult to maintain together with the relevance intuitions. Maintaining the relevance intuitions seems to require that the models allow for the possibility that somewhere, things can, in a sense, "go awry". We can see this playing out in the basic Routley-Meyer models. In Routley-Meyer models, there can be points at which logical truths fail. Putting the point in terms of ubiquity, there must, in general, be points witnessing a failure of ubiquity for logical truths. Were there not such points, then $A \rightarrow(B \rightarrow B)$ and its kin exemplifying irrelevance would be valid. Similarly, there have to be points that, in a sense, get things wrong with respect to what actually is the case. That is the diagnosis of the problem with ubiquity. I will now turn to the issue of interpreting the RMS-frames.

\subsection{Interpreting the Frames}

The picture of actuality presented by these frames departs from the more familiar classical two-dimensional picture according to which one of the worlds in the model is picked out as the actual one. In contrast, in the RMS-frames, different points have candidates, potentially many candidates, they take to be actual, and, in general, there is no distinguished actual point in a frame. Sense can be made of this idea by appealing to a standard interpretation of points in Routley-Meyer frames: points as situations. ${ }^{36}$

The standard interpretation of Routley-Meyer frames takes the points to be situations. ${ }^{37}$ On this interpretation, the points are, or can be, partial and relatively localized things..$^{38}$ It makes sense that there would be a range of options, as situations carry information about what is actual, which can fall short of pinning down a unique situation as the actual world. 39 It would be surprising if a restricted, partial situation carried information sufficient to pin down a situation uniquely as the actual one. That one situation is actual does not rule out another situation,

36. One might also take the points to be bodies of information, as is done Urquhart (1972) or by some work on channel theory, e.g., Mares (1996). Urquhart (1972) deals with operational models, rather than Routley-Meyer models, but the interpretation offered there is natural in the context of Routley-Meyer models. The discussion below carries over fairly readily to this interpretation.

37. See Mares $(1996 ; 2004 ; 2008 ; 2017)$ for more on the interpretation of Routley-Meyer frames in terms of situations.

38. They can be inconsistent as well, although that will not feature in the discussion here.

39. That points can fail to be fully determinate is one of the motivations of possibility semantics. In possibility semantics, the points can be partial, but are consistent, and there is a refinement relation on them. See Humberstone (1981), Holliday (2014), and Benthem, Bezhanishvili, and Holliday (2017) for more on possibility semantics. 
dealing with other matters, from also being actual. Situations can, and often are, parts of a world with other, distinct situations. The situation containing only my office and the situation containing only my apartment are distinct and both are parts of the same world. There is a common situation that contains them both, but there need not be a single situation that contains everything that is, intuitively, in the same world.

Different situations can contain different putative facts about necessity, and they can also contain different putative facts about what is actual. Modal facts in a situation are, perhaps, less concrete than other sorts of facts, such as the weather in Melbourne, but they do not appear to pose any greater philosophical hurdles than contradictory and impossible situations. There are no great difficulties making philosophical sense of RMS-frames, so I will now canvas some options for logics of actuality.

\subsection{Logics of Actuality}

A natural question is what the logic of actuality is, in the context of relevant logics. Nothing said so far pins down a particular logic as the obvious best choice, and there appears to be room for reasonable disagreement. There are, however, a few natural candidates, given a base relevant logic L. One logic is L.MDDc, which adds to the base logic $L$ the axioms $(\mathbb{A} \wedge) \mathbb{A} A \wedge \mathbb{A B} \rightarrow \mathbb{A}(A \wedge B)$, (D) $\mathbb{A} \sim A \rightarrow \sim \mathbb{A} A$, (Dc) $\sim \mathbb{A} A \rightarrow \mathbb{A} \sim A$, and the rule $(\mathrm{RM}) A \rightarrow B \Rightarrow \mathbb{A} A \rightarrow \mathbb{A B}$. In this logic, the actuality operator commutes with the truth-functional connectives, counting negation as such for the moment, just as it does in the classical S5A.

A second candidate logic is $L . M D D c U$, the result of adding $(U), \mathbb{A}(\mathbb{A} A \rightarrow A)$, to L.MDDc. This logic adds the analog of the remaining axiom for actuality from the presentation of Crossley and Humberstone's axiomatization of S5A. This captures the axioms that are arguably distinctive of the actuality operator there. It is, however, not clear that recapturing Crossley and Humberstone's axioms is a desirable target for the relevant logician, as will be considered next. Because of this, I will not consider further extensions of L.MDDcU here.

There is one other natural candidate logic of actuality. Part of the reason for adopting (D) and (Dc) is to recover some of the classical behavior of the actuality operator. It appears to be open to the relevant logician to reject any such recovery as a requirement on the logic, and so to reject any of the additional axioms for the actuality operator, including (D) and (Dc). The interpretation of the frames permits the situations to be so confused as to force an even starker departure from the classical logical behavior of actuality, such as negation no longer commuting with the actuality operator. After all, the frame conditions for the axioms, especially (FDc), (FU), and (FK), are not particularly intuitive or compelling. The idea being that if the situations are permitted to disagree about what is actual, there is not much motivation for requiring the sort of 
coordination needed to validate, say, (Dc). The resulting logic of actuality would be the comparatively weak L.M, which adds only ( $\mathbb{A} \wedge$ ) and $(R M)$ to $L$.

To summarize, three options stand out as natural candidates for logics of actuality. The first is L.MDDc, matching the classical behavior of actuality in the sense of commuting with the truth-functional connectives. The second option is L.MDDcU, which contains axioms that are arguably distinctive of an actuality operator. The third is L.M, motivated by rejecting the logical behavior of actuality in classical modal logic as a guide for the logical behavior of actuality in the present setting and so dropping the additional frame conditions. There is room for disagreement, but the matter will not be settled here. Instead, I will consider using RMS-frames for metaphysical theorizing to explore the consequences for two debates, to which I now turn.

\subsection{Issues in Metaphysics}

In this subsection, I will explore some of the potential consequences of this work for two areas in metaphysics: the indexical-absolute debate in theories of actuality, and the actualism-possibilism debate in ontology.

The Lewisian indexical view of actuality says that "actually" is indexical.40 Each world is actual relative to itself. In contrast to this view, there is the absolute view of actuality, according to which there is only one actual world, which has the distinguishing property of being actual..$^{41}$ From the argument of $\S 4$, it appears that the relevant logician cannot hold an absolute view of actuality, at least if the metaphysics is to have a straightforward connection to the semantics.

We appear to have reached a metaphysical conclusion, that the absolute conception of actuality is untenable for the relevant logician, based only on logical considerations. One might think that a position on the metaphysics of actuality is not something that is logic's business, to adapt the earlier quotation from Slaney. There are, I think, two ways the proponent of absolute actuality and relevant logic may respond. The first is to decouple the truth condition for the "actually" operator from the metaphysics of actuality, and so adopt a truth condition different from that of $\S 4$. In $\$ 6.4$, I will introduce some machinery that may be in harmony with this idea. The second, following Girard and Weber (2015), is perhaps more radical. This response rejects the Routley-Meyer frames presented here on the grounds that they present a distorted picture of the non-classical reality. The alleged problem for the absolutist conception of actuality would, on this view, be diagnosed as an artifact of the Routley-Meyer frames being developed against the background of classical logic. The response proposes that a thoroughly relevant, or more generally non-classical, semantics and metaphysics of actuality

40. See Lewis (1970) and Lewis (1986: 92-96). For discussion, see Adams (1974), Gale (1989), Stephanou (2010), and Davis (2013).

41. This is the simple property view of Adams (1974). 
be developed in a non-classical metatheory, permitting one to adopt the double indexing truth conditions of $\S 4$ for "actually" without the subsequent irrelevancies arising..$^{2}$ The success of that response will have to await the development of the suggested thoroughgoing non-classical model theory that makes good on the suggestion.

The frames have an affinity with the indexical view that is worth comment. As with the indexical view, in the RMS-frames there is no absolute property of being the actual world. Indeed, each point can take itself to be actual. The indexical view is not, however, a perfect fit for these frames. While it is open for each point to take itself to be actual, the frames do not require that each point does take itself to be actual. Additionally, the indexical view brings with it a uniqueness claim: Relative to a world, there is exactly one actual world. This does not hold in the RMS-frames, as the $S$ relation need not be functional. 43 This need not interfere with the validity of $\mathbb{A}(\mathbb{A} A \leftrightarrow A)$, which is a plausible formal gloss on the idea that at the actual world, truth and actual truth coincide. This axiom can be taken on board if desired. While there is an initial affinity between the indexical view of actuality and the RMS-frames, there appears to be room for a distinctive non-classical view of actuality for the relevance-inclined metaphysician.

There may be a lingering sense that the RMS-frames depart too much from familiar conceptions of actuality, that admitting multiple candidates for actuality is a bridge too far, giving up on actuality altogether. One might try to bring the frames closer to the indexical conception of actuality by requiring that $S$ be functional, so that each point has a unique point it takes to be actual. Such frames would obey the condition

$$
\text { (SFun) } \forall x \forall y \forall z(S x y \wedge S x z \Rightarrow y=z) .44
$$

This condition can be expressed in the object language, provided boolean negation, $\neg$, is in the language. Boolean negation has the following truth condition.

- $a \Vdash \neg A$ iff a $\forall$ A

For many relevant logics, one can add boolean negation conservatively, meaning that the addition of boolean negation brings with it no new theorems that lack

42. See Weber, Badia, and Girard (2016) for the development of a related idea with respect to truth tables.

43. Sylvan (1988) denies the uniqueness of the actual world, which he views as an unstated, and false, presupposition. Thanks to an anonymous referee for drawing my attention to this article.

44. (SFun) does not on its own require that every point have an S-successor, just that there is at most one such successor. Every point having an S-successor, or seriality, can be had by adopting (FD). 
that connective. 45 Boolean negation does not cohere well with the philosophical motivations for relevant logics, since it generates ubiquitous truths, such as $p \vee \neg p$, which lead to irrelevancies like $q \rightarrow(p \vee \neg p)$, as well as other examplars of irrelevance, such as $(p \wedge \neg p) \rightarrow q$. It would be unfortunate if the relevant logician, in order to talk about the actual, were forced to adopt boolean negation. ${ }^{4}$ For this reason, it seems best to reject (SFun) as a requirement on frames for actuality. There may be other ways of capturing something of the spirit, if not the letter, of (SFun) that are more palatable to the relevant logician, although there is a further question of whether they mesh with an indexical view of actuality.

Another issue in metaphysics for which there appears to be a distinctively relevant approach is actualism, the view according to which, roughly, everything that exists is actual. 47 The opposing view, possibilism, says that not everything that exists is actual. Leaving aside alethic modals for a moment, a question can arise for actualism in the present setting: What is an actual object? ${ }^{48}$ I will briefly look at how this question arises and how the present setting allows for some distinctively non-classical responses.

To address the question, we will suppose that the RMS-frames have a domain $D_{a}$ of objects for each point $a$, which are not required to all be identical but are such that if $\mathrm{a} \leq \mathrm{b}$ then $\mathrm{D}_{\mathrm{a}} \subseteq \mathrm{D}_{\mathrm{b}}$. To make things a bit more concrete, let us suppose that we have a point, $a$, with the domain $D_{a}=\left\{0_{1}, o_{2}\right\}$. Let us also suppose that the only option for actuality at $a$ is $b$, so Sab and a bears $S$ to nothing else, and that the domain $D_{b}=\left\{o_{1}, o_{2}, o_{3}\right\}$. There is a divergence between the objects at actuality, namely $b$, and the objects existing here at $a$. The object $o_{3}$ is plausibly actual, albeit located modally elsewhere.

The question can be sharpened a bit. Let us alter the scenario by adding two additional points, $c$ and $d$, whose domains are $D_{c}=\left\{o_{1}, o_{2}, o_{4}\right\}$ and $D_{d}=\left\{o_{1}, o_{2}\right\}$, and assuming additionally that $\mathrm{Sac}$ and Sad. 49 The status of $\mathrm{o}_{3}$, and also $\mathrm{o}_{4}$, as

45. See Restall (2000: 306-307). Conservative extension with boolean negation is subtle when there are modal operators in the language, although it can be obtained, as shown by, e.g., Meyer and Mares (1993) and Mares and Tanaka (2010). See Mares (2000) for an example of the addition of boolean negation not yielding a conservative extension.

46. For a similar sentiment regarding boolean negation, albeit in the context of quantification, see Mares and Goldblatt (2006: 183).

47. This formulation is from Menzel (2018), and for references in this active area, see the citations therein. I would like to thank an anonymous referee for suggesting this idea.

48. There are subtleties about quantification in relevant logics that I will bracket here. See Fine (1988), Mares and Goldblatt (2006), Priest (2008: Ch. 24), and Logan (2019) for more on quantification in relevant logics. As far as I am aware, there has not been any investigation of quantified modal relevant logics, at least not in the sense of quantified relevant logics with primitive alethic modal operators. One can treat implications with a fixed antecedent, $A \rightarrow$, as a kind of modal operator, $[A]_{\perp}$, which is sufficient to generate many of the salient issues.

49. An example of the desired set up is a B.MDDc frame that has 8 points, $K=$ $\left\{a, b, c, d, a^{*}, b^{*}, c^{*}, d^{*}\right\}, N=\{a\}, d \leq c, d \leq b, c^{*} \leq d^{*}, b^{*} \leq d^{*}$, the required reflexive pairs for 
actual or merely possible is murky. One tempting response is to say that the actual objects are just those that exist in all candidates for actuality, which are just $o_{1}$ and $o_{2}$ here, with everything else non-actual. Things may not coincide so neatly with the domain at the home point a of this scenario. Let us instead suppose $D_{d}=\left\{o_{1}\right\}$, leaving the rest as is. The tempting response then leads to the conclusion that only $o_{1}$ is an actual object, leaving $o_{2}$ out in the cold of the non-actual, despite it being in the domain $\mathrm{D}_{\mathrm{a}}$ of the home point, $\mathrm{a}$, and leaving $\mathrm{o}_{3}$ and $\mathrm{o}_{4}$ as non-actual, even though they are both in domains of candidates for actuality. The latter consequence makes the initially tempting response slightly less tempting.

To motivate an alternative response, note that the points are generally partial. They need not settle everything, and they can permit much indeterminacy. It is natural, then, to permit being an actual object to be indeterminate, for something to be neither actual nor non-actual. In the second scenario above, it may be better to say that it is indeterminate whether $\mathrm{o}_{3}$ and $\mathrm{o}_{4}$ are actual objects, while $o_{1}$ and $\mathrm{o}_{2}$ are actual objects. On this response, being actual, at a point, is being in the domains of all candidates for actuality at that point, and being non-actual is being excluded from all such domains, with it being indeterminate whether an object is actual if it is in some but not all such domains.

Considerations dual to those of the indeterminacy approach lead to an overdetermination approach. One could offer a theory that takes some objects to be both actual and non-actual. Such a view would say that to be actual is to be in the domain of some candidate for actuality, and to be non-actual is to fail to be in the domain of some actual candidate. On this proposal, in the second scenario above, $\mathrm{o}_{1}, \mathrm{o}_{2}, \mathrm{o}_{3}$, and $\mathrm{o}_{4}$ are all actual with $\mathrm{o}_{3}$ and $\mathrm{o}_{4}$ being non-actual as well.

What is an actual object? In the classical setting, the answer to the question is, plausibly, whatever exists at the actual world. On the relevant logical view of actuality developed here, it is not correct, in general, to take there to be a unique actual point. The potential for there to be multiple candidates for actuality then has ramifications for what counts as an actual object. The candidates for actuality may have different domains, which opens up the option that some objects are neither actual nor non-actual, and that some objects are both actual and non-actual.

There are complications that arise for actualism on the present view. What about for possibilism? Since possibilism is not as restrictive as actualism, it is not clear that there are distinctive problems for possibilism that arise on the present approach. However, to the extent that there are issues arising for the notion of being an actual object, there will be a blurring of the notion of a merely possible object, which opens up space for other sorts of ontological views. Further development of these ideas will require bringing in quantifiers and alethic

$\leq, \mathrm{Sad}, \mathrm{Sac}, \mathrm{Sab}, \mathrm{Sdd}, \mathrm{Sdc}, \mathrm{Sdb}, \mathrm{Scd}, \mathrm{Scc}, \mathrm{Sbd}, \mathrm{Sbb}, \mathrm{Sa}^{*} \mathrm{~d}^{*}, \mathrm{Sd}^{*} \mathrm{~d}^{*}, \mathrm{Sc}^{*} \mathrm{~d}^{*}$, and $\mathrm{Sb}^{*} \mathrm{~d}^{*}$. 
modalities, as well as a thorough reckoning with more precise formulations of actualism and possibilism, which are beyond the scope of this paper. I will now make some connections to other work in two-dimensional logic.

\subsection{Further Two-Dimensional Connections}

The RMS-frames considered in this paper have one binary accessibility relation, $S$, for interpreting actuality. The conditional is an intensional operator, so there are ways to shift points enough to give the actuality operator something to do. Yet, part of the point of introducing actuality, at least in the work of Crossley and Humberstone (1977), is to be able to express claims involving alethic modals, such as possibility and necessity, that interact with actuality. Indeed, they provide some natural language sentences whose proper formalization appears to require alethic modals and an actuality operator.

Suppose we follow suit and add a necessity operator, $\square$, to the language, interpreted in the frames by a new binary relation, $\mathrm{T}$, with conditions in place to ensure the Heredity Lemma can be proven. Let us call such frames RMST-frames. The basic logic of $\square$ on RMST-frames will obey ( $\square \wedge$ ) and ( $\square$ RM), analogs for necessity of the similarly named principles for $\mathbb{A}$.

Without further conditions imposed on the RMST-frames, there will be few substantive, valid interaction principles between $\mathbb{A}$ and $\square$. There will, in fact, be a fair bit of latitude with respect to interaction principles, because the two operators are interpreted by distinct accessibility relations. It is possible to have $\square A \rightarrow \mathbb{A} A$ valid without also having $\mathbb{A} A \rightarrow \square \mathbb{A} A$, for example in B.MDDc frames augmented with another binary accessibility relation..$^{\circ}$

Crossley and Humberstone say that $\mathbb{A} A \rightarrow \square \mathbb{A} A$ may strike some readers as counter-intuitive, and they go on to point out that its validity "arises from the fact that it is one and the same world that counts as the actual world, for every world in the model." 51 A similar fact in the relevant analog of their models, RMg-models, underpinned the Ubiquity Lemma. Crossley and Humberstone use the validity of the axiom to motivate the fixedly operator, $\mathbb{F}$, an operator that shifts the model by changing what the actual world is. They define the relation 'being a variant of $^{\prime}, \simeq$, on pairs of Kripke models $M$ and $M^{\prime}$ with distinguished worlds, so that $M \simeq M^{\prime}$ iff $M$ and $M^{\prime}$ differ at most by what world is distinguished as actual. The truth condition is

- $M, g, w \Vdash \mathbb{F A}$ iff for all $M^{\prime} \simeq M, M^{\prime}, g^{\prime}, w \Vdash A$.

While the particular motivation for such an addition, namely the counterintuitive axiom, is absent in the relevant logical setting, I will note that it is not clear how

50. Finding a countermodel is left to the reader.

51. Crossley and Humberstone (1977: 17) 
one would incorporate $\mathbb{F}$ in the RMS-frames. The fixedly operator is important in discussions of two-dimensional semantics. Davies and Humberstone (1980) gloss the compound operator FAA as expressing the deep necessity of Evans (1979)..$^{2}$ That operator expresses truth at all the pairs $\langle w, w\rangle$, which is sometimes known as the diagonal proposition and is connected to a priori truth. 53 Given the importance of these notions in contemporary metaphysics, it would be good for the relevant logician, or the metaphysician sympathetic to some of the guiding ideas behind relevant logics, to have something to say on this front.

Considerations of "fixedly" prompt an idea for potentially salvaging the double indexing approach found wanting in $\$ 4$. Rather than use the simple RMgframes, we will instead equip RM-frames with an additional ternary relation, $\mathrm{U}$, where Uabc is interpreted as saying: given that $\mathrm{a}$ is actual, $\mathrm{b}$ takes $\mathrm{c}$ to be actual. 54 Call these RMU-frames.

RMU-frames provide two perspectives on actuality. Under the supposition that Uabc, a is considered to be actual, which one can take as stipulating that a is part of how things actually are. This is a global perspective on actuality. Given that $a$ is actual, or is considered as actual, the situation $c$ is actual from the perspective of $b$. This is a local perspective on actuality, from the point of view of the situation $b$. The global perspective, a's being actual, has a bearing on the local perspective, what information the situation $b$ supports, namely that it supports, perhaps erroneously, c's being actual. Were some other situation considered actual, such as $\mathrm{d}, \mathrm{b}$ may not take $\mathrm{c}$ to be actual. This might be the case, for example, if the $a$ and $d$ have different laws of nature or one contains $X Y Z$ and the other $\mathrm{H}_{2} \mathrm{O}$. If certain features of situations considered as actual settle the way laws work or settle certain matters of meaning, then this will, plausibly, affect what situations $b$ takes as candidates for being actual.

Formulas are evaluated in RMU-models relative to pairs of points, a point of evaluation and a distinguished actual point. We can give the following truth conditions for $\mathbb{A}$ and $\mathbb{F}$, with the other connectives carrying over straightforwardly.

- $\mathrm{a}, \mathrm{b} \Vdash \mathbb{A} A$ iff for all $c \in K, \mathrm{Uabc} \Rightarrow \mathrm{a}, \mathrm{c} \Vdash \mathrm{A}$.

- $a, b \Vdash \mathbb{F} A$ iff for all $c \in K, c, b \Vdash A .55$

Additional conditions need to be imposed on the $U$ relation to ensure that the Heredity Lemma holds. It is plausible that RMU-frames could avoid the problems

52. See Davies (2004) and Chalmers (2004) for discussion.

53. For the philosophical importance of the diagonal proposition, see Stalnaker (1999), among others. For application of this idea in natural language semantics, see Fusco (2015). See Restall (2012) for development of related two-dimensional ideas in a proof-theoretic setting.

54. I would like to thank Greg Restall for the initial suggestion of using an additional ternary relation.

55. It seems plausible that quantification over $\mathrm{N}$, or perhaps a different set of points, rather than $\mathrm{K}$, may be more appropriate for the truth condition for $\mathbb{F}$. 
with double indexing canvassed in $\S 4$, as what a point takes to be actual will depend on an additional parameter, namely the point distinguished as actual, while also providing a way to define an analog of diagonal propositions. Indeed, we can take the RMS-frames to be defined from RMU-frames, by taking Sab to be defined as $\mathrm{Ucab}$, for some distinguished $\mathrm{c} \in \mathrm{K}$. Exploration of RMU-frames and their adequacy will, however, be left to future work.

\subsection{Future Directions}

I will close by suggesting two additional directions for future work. One direction is towards hybrid logic..$^{6}$ In hybrid logic, there are devices for talking about particular worlds, the nominals, as well as ways of binding nominals to particular worlds, the binders. These devices allow one to simulate quantification over worlds and obtain a kind of identity predicate on worlds. This specificity is the sort that generated the problems with ubiquity indicated above, which suggests that a relevant logical approach to hybrid logic will require a large departure from the familiar classical theory. The second direction goes to work on temporal logic, such as Kamp (1971). The operator "actually" of modal logic bears many similarities to "now" of temporal logic. This suggests that many of the issues canvassed here will reappear in relevant temporal logics and related views in the philosophy of time. Whether the same sort of responses will be plausible will have to await the development of the relevant theories.

\section{Appendix: Derivability, Soundness, and Completeness}

In earlier sections, several axioms and frame conditions were considered. In this section, I will prove some of the results claimed, including derivability, soundness, and completeness. I will begin with some basic facts about derivability.

\subsection{Derivability}

Fact 1. Let $\mathrm{L}$ be a logic extending $\mathrm{B}$ with additional axioms or rules. Then the following hold.

$$
\begin{aligned}
& \text { If } \vdash_{\mathrm{L}} \mathrm{A} \rightarrow \mathrm{B} \text { and } \vdash_{\mathrm{L}} \mathrm{B} \rightarrow \mathrm{C} \text {, then } \vdash_{\mathrm{L}} \mathrm{A} \rightarrow \mathrm{C} . \\
& \text { If } \vdash_{\mathrm{L}} \mathrm{A} \rightarrow(\mathrm{B} \rightarrow \mathrm{C}) \text { and } \vdash_{\mathrm{L}} \mathrm{C} \rightarrow \mathrm{D} \text {, then } \vdash_{\mathrm{L}} \mathrm{A} \rightarrow(\mathrm{B} \rightarrow \mathrm{D}) . \\
& \text { If } \vdash_{\mathrm{L}} \mathrm{A} \rightarrow(\mathrm{B} \rightarrow \mathrm{C}) \text { and } \vdash_{\mathrm{L}} \mathrm{D} \rightarrow \mathrm{B} \text {, then } \vdash_{\mathrm{L}} \mathrm{A} \rightarrow(\mathrm{D} \rightarrow \mathrm{C}) .
\end{aligned}
$$

Proof. For the first, we argue as follows. Assume the derivability of $A \rightarrow B$ and $B \rightarrow C$. From $(R 3),(B \rightarrow C) \rightarrow(A \rightarrow C)$, and then from $(R 1) A \rightarrow C$.

56. For more on hybrid logic, see Braüner (2017) for an excellent overview of the area, the citations therein, and the earlier citations of work on non-classical hybrid logic. I would like to thank an anonymous referee for making this suggestion. 
For the second, we argue as follows. Assume the derivability of $A \rightarrow(B \rightarrow C)$ and $C \rightarrow D$. From $(R 4)$, we have $(B \rightarrow C) \rightarrow(B \rightarrow D)$, from which $A \rightarrow(B \rightarrow D)$ follows using the previous proof.

For the third, the argument is largely the same as the preceding, using (R3) rather than (R4).

With these basic facts in place, I will prove two lemmas about actuality logics.

Lemma 4. L.MDDc has as theorems $\sim \mathbb{A} \sim A \leftrightarrow \mathbb{A} A$ and $\mathbb{A}(A \vee B) \leftrightarrow \mathbb{A} A \vee \mathbb{A B}$.

Proof. For the first claim, we argue as follows for one direction. By (D), $\mathbb{A} \sim A \rightarrow$ $\sim \mathbb{A} A$. Then, $\sim \sim \mathbb{A} A \rightarrow \sim \mathbb{A} \sim A$, from (R5), and so $\mathbb{A} A \rightarrow \sim \mathbb{A} \sim A$, from $(A 9)$ and fact 1.

For the other direction, we have $\sim \mathbb{A} A \rightarrow \mathbb{A} \sim A$ from $(\mathrm{Dc})$. As before, we have $\sim \mathbb{A} \sim A \rightarrow \sim \mathbb{A} A$ from $(\mathrm{R} 5)$, whence $\sim \mathbb{A} \sim A \rightarrow \mathbb{A} A$ from $(\mathrm{A} 9)$ and the previous lemma.

The second claim unpacks to $\mathbb{A} \sim(\sim \mathrm{A} \wedge \sim \mathrm{B}) \leftrightarrow \sim(\sim \mathbb{A} A \wedge \sim \mathbb{A} B)$. This can be obtained using $(R M),(\mathbb{A} \wedge),(R 5)$ and the preceding equivalence from $(\sim A \wedge$ $\sim B) \leftrightarrow(\sim A \wedge \sim B)$.

Lemma 5. If (B7), (Dc), and (U) are theorems of $L . M X$, then so is $\mathbb{A}(A \rightarrow \mathbb{A} A)$.

Proof. By (B7), we have $(\mathbb{A} \sim A \rightarrow \sim A) \rightarrow(\sim \sim A \rightarrow \sim \mathbb{A} \sim A)$, as well as $\sim \mathbb{A} \sim A \rightarrow \mathbb{A} A$, by lemma 4. We then have $(\mathbb{A} \sim A \rightarrow \sim A) \rightarrow(A \rightarrow \mathbb{A} A)$, from fact 1 . From axiom $(\mathrm{RM})$, we obtain $\mathbb{A}(\mathbb{A} \sim A \rightarrow \sim A) \rightarrow \mathbb{A}(A \rightarrow \mathbb{A} A)$, and $\mathbb{A}(\mathbb{A} \sim A \rightarrow \sim A)$ from $(U)$, so $\mathbb{A}(A \rightarrow \mathbb{A} A)$, by $(\mathrm{R} 1)$.

\subsection{Soundness}

In this subsection, I will prove some lemmas leading to a proof of soundness for a range of logics. I will show that for each axiom, that axiom is valid in a class of frames if all the frames in the class obey the condition corresponding to the axiom. From this, it follows that a particular logic of actuality is sound with respect to the associated class of frames.

Lemma 6. The axiom (Dc) $\sim \mathbb{A} A \rightarrow \mathbb{A} \sim \mathrm{A}$ is valid in a class of RMS-frames if that class obeys the condition (FDc) $\forall x \forall y \forall z\left(S x^{*} y \wedge S x z \Rightarrow y^{*} \leq z\right)$.

Proof. Suppose the class of frames obeys the condition (FDc). Let $M$ be a model on a frame in the class and $a \in K$. Suppose $a \Vdash \sim \mathbb{A} A$ but $a \| \mathbb{A} \sim A$. Then it follows that for some $c$, Sac and $c \| \forall \sim A$. So, $c^{*} \Vdash A$. By assumption, $a^{*} \forall \mathbb{A} A$, so for some $\mathrm{b}, \mathrm{Sa}^{*} \mathrm{~b}$ and $\mathrm{b} \| \mathrm{A}$. The condition implies that $\mathrm{b}^{*} \leq \mathrm{c}$, so $\mathrm{c}^{*} \leq \mathrm{b}$, whence $\mathrm{b} \Vdash \mathrm{A}$, contradicting the earlier conclusion. 
Lemma 7. The axiom (U) $\mathbb{A}(\mathbb{A} A \rightarrow A)$ is valid in a class of RMS-frames if that class obeys the condition (FU) $\forall x \in N, \forall y(S x y \Rightarrow \forall z \forall u(R y z u \Rightarrow S z u))$.

Proof. Suppose a class of frames obeys the condition (FU) but (U) is not valid. Then there is a model on a frame in the class with a point $a \in N$ such that $a \| \mathbb{A}(\mathbb{A} A \rightarrow A)$. So for some $b$, Sab and $b \forall \mathbb{A} A \rightarrow A$. Therefore, there are $c, d$ such that Rbcd, $c \Vdash \mathbb{A} A$, and $d \forall A$. The frame condition implies Scd, whence $\mathrm{d} \Vdash A$, which is a contradiction.

Lemma 8. The axiom (Uc) $\mathbb{A}(A \rightarrow \mathbb{A} A)$ is valid in a class of RMS-frames if the class obeys the condition (FUc) $\forall x \in N, \forall y(S x y \Rightarrow \forall u \forall v(R y u v \Rightarrow \forall z(S v z \Rightarrow u \leq z)))$.

Proof. Suppose the class obeys the condition (FUc) but $\mathbb{A}(A \rightarrow \mathbb{A} A)$ is not valid. Then there is a model $M$ and a point $a \in N$ such that $a \forall \mathbb{A}(A \rightarrow \mathbb{A} A)$. So, there is $a b$ such that $S a b$ and $b \forall A \rightarrow \mathbb{A} A$. Then there are $d$ and e such that Rbde, $\mathrm{d} \Vdash A$, but $e \Vdash \mathbb{A} A$. This implies there is a $c$, Sec and $c \forall A$. The frame condition then implies that $d \leq c$, which by the Heredity Lemma yields $c \Vdash A$.

The preceding lemmas suffice for the following soundness claim. The notation $\mathcal{F}(\mathrm{L} . \mathrm{MX})$ will be adapted to RMS-frames satisfying the frame conditions associated with the axioms and rules of L.MX.

Theorem 2 (Soundness). Let the logic $\mathrm{L}$ extend $\mathrm{B}$ with any of the axioms from (B1)(B8), and suppose $\mathrm{X} \subseteq\{(\mathrm{Nec}),(\mathrm{U}),(\mathrm{Uc}),(\mathrm{D}),(\mathrm{Dc}),(\mathrm{K}),(4)\}$. Then, $\vdash_{\mathrm{L} . \mathrm{MX}}$ A only if $\models_{\mathcal{F}(\text { L.MX) }}$ A.

\subsection{Completeness}

With soundness in hand, I will now turn to completeness. The approach of this section is to use the Henkin-style canonical model construction familiar from work in the area, leaning on the particular developments of Fuhrmann (1990) and Restall (2000). For a given logic, one defines a frame and then shows that it satisfies all the conditions needed to be a frame for the logic. Then, one shows that the evaluation clauses work as expected, and so one can define a model on the frame to refute the target non-theorem. At the end of this subsection, I will sketch a proof demonstrating that the logics of actuality from RMS-frames respect a necessary condition on relevance, Belnap's variable sharing constraint.

It follows from the work of Fuhrmann (1990) that, for a logic $L$ extending $B$ with axioms from (B1)-(B8), L.MX is sound and complete with respect to $\mathcal{F}($ L.MX), where $X \subseteq\{(\mathrm{Nec}),(4),(\mathrm{D}),(\mathrm{K})\}$. I will, then, focus on axioms not already covered. In the interest of making this paper more self-contained, I will reproduce some of the standard definitions. 
Definition 2. A set $\mathrm{X}$ of formulas is an L-theory iff

- if $\mathrm{A} \in \mathrm{X}$ and $\vdash_{\mathrm{L}} \mathrm{A} \rightarrow \mathrm{B}$, then $\mathrm{B} \in \mathrm{X}$, and

- if $\mathrm{A} \in \mathrm{X}$ and $\mathrm{B} \in \mathrm{X}$, then $\mathrm{A} \wedge \mathrm{B} \in \mathrm{X}$.

A theory $\mathrm{X}$ is prime iff $\mathrm{A} \vee \mathrm{B} \in \mathrm{X}$ implies $\mathrm{A} \in \mathrm{X}$ or $\mathrm{B} \in \mathrm{X}$.

$A$ theory $\mathrm{X}$ is L-regular iff $\mathrm{L} \subseteq \mathrm{X}$.

For all the defined concepts, when $L$ is clear from context, it will be dropped.

The canonical model $M$ for the logic L.MX will be defined as follows, where for sets of formulas $X, X^{-\mathbb{A}}=\{A: \mathbb{A} A \in X\}$.

- $K_{M}$ is the set of prime L.MX-theories.

- $\mathrm{N}_{M}$ is the set of L.MX-regular, prime L.MX-theories.

- $R_{M} a b c$ iff $\forall A \forall B(A \rightarrow B \in a \wedge A \in b \Rightarrow B \in c)$.

- $\mathrm{a}^{* M}=\{\mathrm{A}: \sim \mathrm{A} \notin \mathrm{a}\}$.

- $S_{M} a b$ iff $a^{-\mathbb{A}} \subseteq b$.

- $V_{M}(p)=\left\{a \in K_{M}: p \in a\right\}$.

In what follows, the subscript ' $M$ ' will be omitted to avoid clutter.

The canonical frame obeys the required frame condition for RMS-frames,

- $\mathrm{a} \leq \mathrm{b} \Rightarrow \mathrm{Sb} \subseteq \mathrm{Sa} .57$

Further, by standard arguments one can show that it satisfies the conditions required by the base logic L. $5^{8}$ We need to show that the frame underlying the canonical model for the logic L.MX satisfies the frame conditions (FA), for each axiom $(A) \in X$. I will provide proofs for the axioms $(D c),(U)$, and $(U c)$.

Lemma 9. If the logic L.MX contains (Dc), then the canonical frame obeys the condition (FDc).

Proof. Suppose L.MX contains (Dc). Further, suppose $S a^{*} b, S a c$, but not $b^{*} \leq c$. Then there is some $B \in b^{*}$ such that $B \notin c$. Therefore, $A B \notin a$, so $\sim A B \in a^{*}$. Since $\mathrm{a}^{*}$ is a theory and $\vdash_{\mathrm{L} . \mathrm{MX}} \sim \mathbb{A B} \rightarrow \mathbb{A} \sim \mathrm{B}, \mathbb{A} \sim \mathrm{B} \in \mathrm{a}^{*}$. Thus, $\sim \mathrm{B} \in \mathrm{b}$, so $\mathrm{B} \notin \mathrm{b}^{*}$, contradicting the assumption.

Lemma 10. If the logic L.MX contains $(\mathrm{U})$, then the canonical frame obeys the condition (FU).

57. The canonical frame also obeys the optional condition flagged earlier: $\mathrm{Sab} \wedge \mathrm{b} \leq$ $\mathrm{c} \Rightarrow \mathrm{Sac}$.

58. See Routley et al. (1982: Ch. 4).

Ergo $\cdot$ vol. 7, no. $8 \cdot 2020$ 
Proof. Suppose L.MX contains (U). Suppose $a \in N, S a b, R b c d$, but not Scd. Then there is some $B$ such that $A B \in c$ and $B \notin d$. By definition, $A B \rightarrow B \notin b$, so $\mathbb{A}(\mathbb{A B} \rightarrow \mathrm{B}) \notin \mathrm{a}$. But, as $\mathrm{a} \in \mathrm{N}, \mathbb{A}(\mathbb{A B} \rightarrow \mathrm{B}) \in \mathrm{a}$, which is a contradiction.

Lemma 11. If the logic L.MX contains ( $\mathrm{Uc}$ ), then the canonical frame obeys the condition (FUc).

Proof. Suppose L.MX contains (Uc). Suppose $a \in N, S a b, R b c d, S d e$, but not $c \leq e$. Then there is some $B, B \in c$ but $B \notin e$. By definition, $A B \notin d$, so $B \rightarrow \mathbb{A B} \notin b$. Therefore, $\mathbb{A}(B \rightarrow \mathbb{A B}) \notin a$. But, as $\vdash_{\mathrm{L} . \mathrm{MX}} \mathbb{A}(\mathrm{B} \rightarrow \mathbb{A B})$ and $\mathrm{a} \in \mathrm{N}, \mathbb{A}(\mathrm{B} \rightarrow \mathbb{A B}) \in \mathrm{a}$, which is a contradiction.

The frame underlying the canonical model for a logic containing any of the preceding actuality axioms obeys the frame condition for that axiom. It is fairly routine to establish the following.

Lemma 12 (Truth Lemma). In the canonical model for the logic L.MX, for all formulas $A, A \in a$ iff a $\Vdash A$.

Proof. See Fuhrmann (1990) or Restall (2000: Ch. 11).

When the preceding is combined with the results from Fuhrmann (1990), we have the following completeness result.

Theorem 3 (Completeness). Let the logic $\mathrm{L}$ extend $\mathrm{B}$ with any of the axioms from $(\mathrm{B} 1)-(\mathrm{B} 8)$, and suppose $\mathrm{X} \subseteq\{(\mathrm{Nec}),(\mathrm{U}),(\mathrm{Uc}),(\mathrm{D}),(\mathrm{Dc}),(\mathrm{K}),(4)\}$. Then, $\models_{\mathcal{F}(\mathrm{L} . \mathrm{MX})} \mathrm{A}$ only if $\vdash_{\text {L.MX }}$ A.

With the completeness results in hand, I can now argue that the RMS-frames preserve relevance intuitions in the sense that they have the variable sharing property, just as the underlying relevant logics do.

Theorem 4 (Variable sharing). For any of the logics L.MX proved complete above, for all formulas $\mathrm{A}$ and $\mathrm{B}$ not containing $\mathrm{t}$, if $\vdash$ L.MX $\mathrm{A} \rightarrow \mathrm{B}$, then $\mathrm{A}$ and $\mathrm{B}$ share a propositional variable.

Proof. (Sketch) The proof is adapted from proofs that $\mathrm{R}$ has the variable sharing property, as described by Dunn and Restall (2002: §1.6), using the algebraic method of Anderson and Belnap (1975: 252-254), whose Hasse diagram and matrices are in Table 2.59 The designated values are marked + and $t$ is always assigned the value +0 . For the present context, interpret $\mathbb{A}$ as the identity operator. All actuality axioms considered in this paper are designated on all valuations on these matrices, $(\mathrm{Nec})$ preserves the property of being designated, and similarly for all axioms and rules of $\mathrm{R}$.

59. See also theorem 8.54 of Restall (2000: 184) and Priest (2008: 205-206).

Ergo • vol. 7, no. $8 \cdot 2020$ 


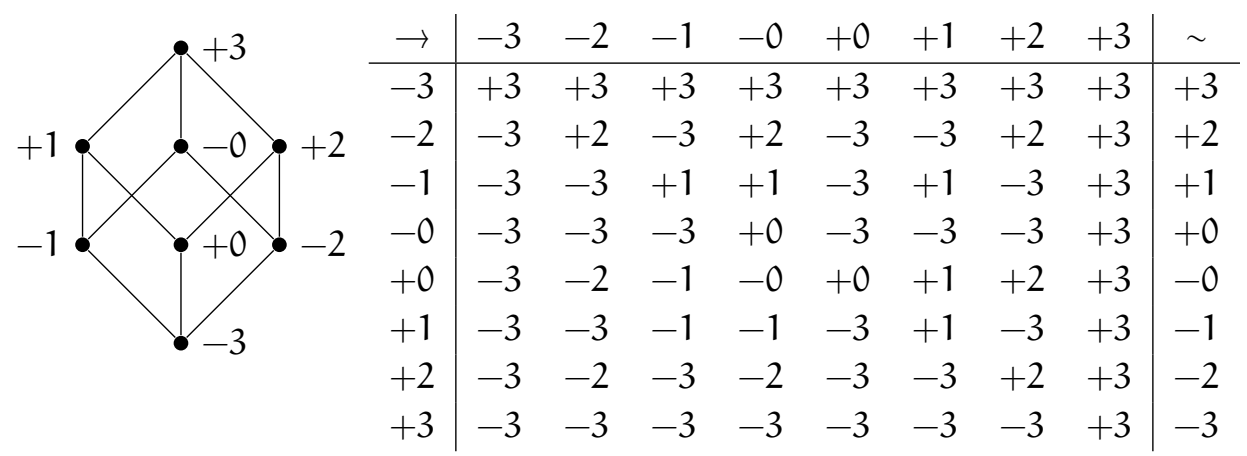

Table 2. Hasse diagram and matrices for the variable sharing property

Suppose that $\vdash_{\text {L.MX }} A \rightarrow B$ for some t-free formulas $A$ and $B$ but $A$ and $B$ do not share a propositional variable. Let $A^{\prime}$ and $B^{\prime}$ be $A$ and $B$, respectively, with all instances of the actuality operator removed. Then $A^{\prime}$ and $B^{\prime}$ take the same values as $A$ and $B$, respectively. Since $A$ and $B$ do not share a propositional variable, neither do $A^{\prime}$ and $B^{\prime}$, so there is a valuation on the matrix such that $A^{\prime} \rightarrow B^{\prime}$ is not designated, which implies that $A \rightarrow B$ is undesignated. Therefore, $A \rightarrow B$ is not provable, contradicting the assumption. As all the relevant logics considered in this paper are sublogics of $\mathrm{R}$, the argument works for all of them.

Thus, we see that the logic of the actuality operator, as delivered by the RMSframes, maintains the necessary condition on relevance, an indication that they at least modestly respect the relevance intuitions with which we started.

\section{Acknowledgements}

I would like to thank Greg Restall, Rohan French, Dave Ripley, Lloyd Humberstone, Melissa Fusco, Fabio Lampert, John Bigelow, and the audience at the Melbourne Logic Seminar for comments and discussion that have greatly improved this paper. I would also like to thank the anonymous referees whose feedback significantly improved this paper. This research was supported by the Australian Research Council, Discovery Grant DP150103801.

\section{References}

Adams, Robert Merrihew (1974). Theories of Actuality. Noûs, 8(3), 211-231. https://doi.org/10.2307/2214751

Anderson, Alan Ross and Nuel D. Belnap (1975). Entailment: The Logic of Relevance and Necessity, Vol. I. Princeton University Press. 
Avron, Arnon (1992). Whither Relevance Logic? Journal of Philosophical Logic, 21(3), 243-281. https://doi.org/10.1007/bfoo260930

Beall, Jc, Ross Brady, J. Michael Dunn, A. P. Hazen, Edwin Mares, Robert K. Meyer, ... , Richard Sylvan (2012). On the Ternary Relation and Conditionality. Journal of Philosophical Logic, 41(3), 595-612. https://doi.org/10.1007/ s10992-011-9191-5

van Benthem, Johan, Nick Bezhanishvili, and Wesley H. Holliday (2017). A Bimodal Perspective on Possibility Semantics. Journal of Logic and Computation, 27(5), 1353-1389. https://doi.org/10.1093/logcom/exw024

Bimbó, Katalin (2006). Relevance Logics. In Dale Jacquette (Ed.), Philosophy of Logic (723-789), Vol. 5 of Handbook of the Philosophy of Science. Elsevier.

Blackburn, Patrick and Maarten Marx (2002). Remarks on Gregory's "Actually" Operator. Journal of Philosophical Logic, 31(3), 281-288. https://doi.org/10. 1023/a:1015726824270

Brady, Ross T. (1984). Natural Deduction Systems for Some Quantified Relevant Logics. Logique Et Analyse, 27(8), 355-377.

Braüner, Torben (2006). Axioms for Classical, Intuitionistic, and Paraconsistent Hybrid Logic. Journal of Logic, Language and Information, 15(3), 179-194. https: //doi.org/10.1007/s10849-006-9013-2

Braüner, Torben (2007). Why Does the Proof-Theory of Hybrid Logic Work So Well? Journal of Applied Non-Classical Logics, 17(4), 521-543. https: / / doi.org/ 10.3166/jancl.17.521-543

Braüner, Torben (2011). Intuitionistic Hybrid Logic: Introduction and Survey. Information and Computation, 209(12), 1437-1446. https://doi.org/10.1016/j.ic. 2011.10.001

Braüner, Torben (2017). Hybrid Logic. In Edward N. Zalta (Ed.), The Stanford Encyclopedia of Philosophy (Summer 2017 ed.). Retrieved from https://plato. stanford.edu/archives/sum2017/entries/logic-hybrid/

Braüner, Torben and Valeria de Paiva (2006). Intuitionistic hybrid logic. Journal of Applied Logic, 4(3), 231-255. https://doi.org/10.1016/j.jal.2005.06.009

Chalmers, David J. (2004). Epistemic Two-Dimensional Semantics. Philosophical Studies, 118(1/2), 153-226. https://doi.org/10.1023/b:phil.ooooo19546.17135. eo

Costa, Diana and Manuel A. Martins (2017). Paraconsistency in Hybrid Logic. Journal of Logic and Computation, 27(6), 1825-1852. https://doi.org/10.1093/ $\log \mathrm{com} /$ exwo27

Crossley, John N. and Lloyd Humberstone (1977). The Logic of "Actually". Reports on Mathematical Logic, 8, 11-29.

Davies, Martin (2004). Reference, Contingency, and the Two-Dimensional Framework. Philosophical Studies, 118(1/2), 83-131. https://doi.org/10.1023/b: phil.ooooo19544.39800.50 
Davies, Martin and Lloyd Humberstone (1980). Two Notions of Necessity. Philosophical Studies, 38(1), 1-31. https://doi.org/10.1007/BFo0354523

Davis, Wayne A. (2013). The Semantics of Actuality Terms: Indexical vs. Descriptive Theories. Noûs, 49(3), 470-503. https://doi.org/10.1111/nous.12046

Dunn, J. Michael and Greg Restall (2002). Relevance Logic. In Dov M. Gabbay and Franz Guenthner (Eds.), Handbook of Philosophical Logic (2nd ed., Vol. 6, 1-136). Kluwer.

Evans, Gareth (1979). Reference and Contingency. The Monist, 62(2), 161-189. https: / / doi.org/10.5840/monist197962220

Fine, Kit (1988). Semantics for Quantified Relevance Logic. Journal of Philosophical Logic, 17(1), 27-59. https://doi.org/10.1007/BFo0249674

French, Rohan (2012). An Argument against General Validity? Thought: A Journal of Philosophy, 1(1), 4-9. https://doi.org/10.1002/tht3.1

French, Rohan (2015). In the Mood for $\mathrm{S}_{4}$ : The Expressive Power of the Subjunctive Modal Language in Weak Background Logics. Studia Logica, 103(2), 239-263. https: / / doi.org/10.1007/s11225-014-9557-0

Fritz, Peter (2013). A Logic for Epistemic Two-Dimensional Semantics. Synthese, 190(10), 1753-1770. https://doi.org/10.1007/s11229-013-0260-X

Fritz, Peter (2014). What Is The Correct Logic of Necessity, Actuality and Apriority? The Review of Symbolic Logic, 7(3), 385-414. https:/ / doi.org/10.1017/ S1755020314000136

Fuhrmann, André (1990). Models for Relevant Modal Logics. Studia Logica, 49(4), 501-514. https://doi.org/10.1007/bfo0370161

Fusco, Melissa (2015). Deontic Modality and the Semantics of Choice. Philosophers' Imprint, 15(28), 1-27. Retrieved from http:/ / hdl.handle.net/2027/spo.3521354. 0015.028

Gale, Richard M. (1989). Lewis' Indexical Argument for World-Relative Actuality. Dialogue, 28(2), 289-304. https://doi.org/10.1017/so01221730001578x

Giambrone, Steve (1992). Real Reduced Models for Relevant Logics without WI. Notre Dame Journal of Formal Logic, 33(3), 442-449. https:/ / doi.org/10.1305/ ndjfl/1093634408

Gilbert, David (2016). Two-Dimensional Tableaux. Australasian Journal of Logic, 13(7), 143-170. https://doi.org/10.26686/ajl.v13i7.3894

Gilbert, David R. and Edwin D. Mares (2012). Completeness Results for Some Two-Dimensional Logics of Actuality. Review of Symbolic Logic, 5(2), 239-258. https:/ / doi.org/10.1017/S1755020311000384

Girard, Patrick and Zach Weber (2015). Bad Worlds. Thought: A Journal of Philosophy, 4(2), 93-101. https://doi.org/10.1002/tht3.162

Gregory, Dominic (2001). Completeness and Decidability Results for Some Propositional Modal Logics Containing 'Actually' Operators. Journal of Philosophical Logic, 30(1), 57-78. https://doi.org/10.1023/A:1017579410231 
Hanson, William H. (2006). Actuality, Necessity, and Logical Truth. Philosophical Studies, 130(3), 437-459. https://doi.org/10.1007/s11098-004-5750-8

Hazen, Allen P., Benjamin G. Rin, and Kai F. Wehmeier (2013). Actuality in Propositional Modal Logic. Studia Logica, 101(3), 487-503. https://doi.org/10. 1007/s11225-012-9395-X

Holliday, Wesley H. (2014). Partiality and Adjointness in Modal Logic. In Rajeev Goré, Barteld Kooi, and Agi Kurucz (Eds.), Advances in Modal Logic (Vol. 10, 313-332). College Publications.

Humberstone, Lloyd (1981). From Worlds to Possibilities. Journal of Philosophical Logic, 10(3), 313-339. https://doi.org/10.1007/bfoo293423

Humberstone, Lloyd (2004). Two-Dimensional Adventures. Philosophical Studies, 118(1-2), 17-65. https://doi.org/10.1023/B:PHIL.00ooo19542.43440.d1

Humberstone, Lloyd (2011). The Connectives. MIT Press.

Humberstone, Lloyd (2016). Philosophical Applications of Modal Logic. College Publications.

Kamp, Hans (1971). Formal Properties of "Now". Theoria, 37(3), 227-273. https: //doi.org/10.1111/j.1755-2567.1971.tbooo71.x

Kripke, Saul A. (1963). Semantical Analysis of Modal Logic I: Normal Propositional Calculi. Zeitschrift fur mathematische Logik und Grundlagen der Mathematik, 9(56), 67-96. https://doi.org/10.1002/malq.19630090502

Lampert, Fabio (2018a). Actuality, Tableaux, and Two-Dimensional Modal Logics. Erkenntnis, 83(3), 403-443. https://doi.org/10.1007/s10670-017-9896-o

Lampert, Fabio (2018b). Actuality and the A Priori. Philosophical Studies, 175(3), 809-830. https://doi.org/10.1007/s11098-017-0894-5

Lewis, David (1970). Anselm and Actuality. Noûs, 4(2), 175-188. https://doi.org/ $10.2307 / 2214320$

Lewis, David (1986). On the Plurality of Worlds. Wiley-Blackwell.

Logan, Shay Allen (2019). Notes on Stratified Semantics. Journal of Philosophical Logic, 48(4), 749-786. https://doi.org/10.1007/s10992-018-9493-y

Mares, Edwin D. (1992). Semantics for Relevance Logic with Identity. Studia Logica, 51(1), 1-20. https://doi.org/10.1007/BFo0370329

Mares, Edwin D. (1996). Relevant Logic and the Theory of Information. Synthese, 109(3), 345-360. https://doi.org/10.1007/bfoo413865

Mares, Edwin D. (2000). CE is Not a Conservative Extension of E. Journal of Philosophical Logic, 29(3), 263-275. https://doi.org/10.1023/a:1004731401855

Mares, Edwin D. (2004). Relevant Logic: A Philosophical Interpretation. Cambridge Univeristy Press.

Mares, Edwin D. (2008). General Information in Relevant Logic. Synthese, 167(2), 343-362. https://doi.org/10.1007/s11229-008-9412-9

Mares, Edwin D. (2017). An Informational Interpretation of Weak Relevant Logic and Relevant Property Theory. Synthese. Advance online publication. 
https://doi.org/10.1007/s11229-017-1524-7

Mares, Edwin D. and Robert Goldblatt (2006). An Alternative Semantics for Quantified Relevant Logic. The Journal of Symbolic Logic, 71(1), 163-187. https: //doi.org/10.2178/jsl/1140641167

Mares, Edwin D. and Robert K. Meyer (1993). The Semantics of R4. Journal of Philosophical Logic, 22(1), 95-110. https://doi.org/10.1007/BFo1049182

Mares, Edwin D. and Koji Tanaka (2010). Boolean Conservative Extension Results for Some Modal Relevant Logics. The Australasian Journal of Logic, 8, 31-49. https://doi.org/10.26686/ajl.v8io.1812

Menzel, Christopher (2018). Actualism. In Edward N. Zalta (Ed.), The Stanford Encyclopedia of Philosophy (Summer 2018 ed.). Retrieved from https://plato. stanford.edu/archives/sum2018/entries/actualism/

Meyer, Robert K. and Edwin D. Mares (1993). Semantics of Entailment o. In Peter Schroeder-Heister and Kosta Došen (Eds.), Substructural Logics (239-258). Oxford Science Publications.

Nelson, Michael and Edward N. Zalta (2012). A Defense of Contingent Logical Truths. Philosophical Studies, 157(1), 153-162. https://doi.org/10.1007/ s11098-010-9624-y

Priest, Graham (2006). In Contradiction: A Study of the Transconsistent (2nd ed.). Oxford University Press.

Priest, Graham (2008). An Introduction to Non-Classical Logic: From If to Is. Cambridge University Press.

Read, Stephen (1988). Relevant Logic: A Philosophical Examination of Inference. Basil Blackwell.

Restall, Greg (1999). Negation in Relevant Logics: How I Stopped Worrying and Learned to Love the Routley Star. In Dov Gabbay and Heinrich Wansing (Eds.), What Is Negation? (53-76), Vol. 13 of Applied Logic Series. Kluwer Academic Publishers.

Restall, Greg (2000). An Introduction to Substructural Logics. Routledge.

Restall, Greg (2012). A Cut-Free Sequent System for Two-Dimensional Modal Logic, and Why It Matters. Annals of Pure and Applied Logic, 163(11), 1611-1623. https://doi.org/10.1016/j.apal.2011.12.012

Routley, Richard (1977). Ultralogic as Universal? Relevance Logic Newsletter, 2, 51-89. Reprinted as Appendix in Richard Routley (1980) Exploring Meinong's Jungle and Beyond (893-962). Page numbers are for the reprinted version.

Routley, Richard (1984). The American Plan Completed: Alternative ClassicalStyle Semantics, without Stars, for Relevant and Paraconsistent Logics. Studia Logica, 43(1-2), 131-158. https://doi.org/10.1007/BFoo935746

Routley, Richard and Robert K. Meyer (1972a). The Semantics of Entailment-II. Journal of Philosophical Logic, 1(1), 53-73. https://doi.org/10.1007/BFoo649991 Routley, Richard and Robert K. Meyer (1972b). The Semantics of Entailment- 
III. Journal of Philosophical Logic, 1(2), 192-208. https://doi.org/10.1007/ bfoo650498

Routley, Richard and Robert K. Meyer (1973). The Semantics of Entailment. In Hughes Leblanc (Ed.), Truth, Syntax, and Modality: Proceedings Of The Temple University Conference On Alternative Semantics (199-243). North-Holland.

Routley, Richard, Val Plumwood, Robert K. Meyer, and Ross T. Brady (1982). Relevant Logics and Their Rivals (Vol. 1). Ridgeview.

Schroeter, Laura (2017). Two-Dimensional Semantics. In Edward N. Zalta (Ed.), The Stanford Encyclopedia of Philosophy (Summer 2017 ed.). Retrieved from https://plato.stanford.edu/archives/sum2017/entries/ two-dimensional-semantics/

Sedlár, Igor (2015). Substructural Epistemic Logics. Journal of Applied Non-Classical Logics, 25(3), 256-285. https://doi.org/10.1080/11663081.2015.1094313

Slaney, John K. (1987). Reduced Models for Relevant Logics without WI. Notre Dame Journal of Formal Logic, 28(3), 395-407. https://doi.org/10.1305/ndjfl/ 1093637560

Stalnaker, Robert (1999). Assertion. In Context and Content (78-95). Oxford University Press.

Stephanou, Yannis (2010). The Meaning of "Actually". Dialectica, 64(2), 153-185. https://doi.org/10.1111/j.1746-8361.2010.01222.x

Sylvan, Richard (1988). Radical Pluralism: An Alternative to Realism, Antirealism and Relativism. In Robert Nola (Ed.), Relativism and Realism in Science (253291). Kluwer.

Tennant, Neil (2017). Core Logic. Oxford University Press.

Urquhart, Alasdair (1972). Semantics for Relevant Logics. Journal of Symbolic Logic, 37(1), 159-169. https://doi.org/10.2307/2272559

Weber, Zach, Guillermo Badia, and Patrick Girard (2016). What Is an Inconsistent Truth Table? Australasian Journal of Philosophy, 94(3), 533-548. https://doi. org/10.1080/00048402.2015.1093010

Wehmeier, Kai Frederick (2004). In the Mood. Journal of Philosophical Logic, 33(6), 607-630. https://doi.org/10.1023/b:logi.ooooo46193.69391.18

Zalta, Edward N. (1988). Logical and Analytic Truths that Are Not Necessary. The Journal of Philosophy, 85(2), 57-74. https://doi.org/10.2307/2026992 